\title{
TOPSIS Method Based on the Correlation Coefficient of Interval-Valued Intuitionistic Fuzzy Soft Sets and Aggregation Operators with Their Application in Decision-Making
}

\author{
Rana Muhammad Zulqarnain (D), ${ }^{1}$ Xiao Long Xin $\left(\mathbb{D},{ }^{1}\right.$ Muhammad Saqlain $\left(\mathbb{D},{ }^{1}\right.$ \\ and Waseem Asghar Khan ${ }^{2}$ \\ ${ }^{1}$ School of Mathematics, Northwest University Xi'an, Xi'an, China \\ ${ }^{2}$ Department of Mathematics, Faculty of Sciences AlZulf, Majmaah University, Majmaah 11952, Saudi Arabia \\ Correspondence should be addressed to Xiao Long Xin; xlxin@nwu.edu.cn
}

Received 3 October 2020; Revised 16 November 2020; Accepted 18 December 2020; Published 29 January 2021

Academic Editor: Feng Feng

Copyright (C) 2021 Rana Muhammad Zulqarnain et al. This is an open access article distributed under the Creative Commons Attribution License, which permits unrestricted use, distribution, and reproduction in any medium, provided the original work is properly cited.

\begin{abstract}
The correlation coefficient between the two parameters plays a significant part in statistics. Furthermore, the exactness of the assessment of correlation depends upon information from the set of discourses. The data collected for various statistical studies are full of ambiguities. The idea of interval-valued intuitionistic fuzzy soft sets is an extension of intuitionistic fuzzy soft sets that is used to express insufficient evaluation, uncertainty, and anxiety in decision-making. Intuitionistic fuzzy soft sets consider two different types of information, such as membership degree and nonmembership degree. In this paper, the concepts and properties of the correlation coefficient and the weighted correlation coefficient of interval-valued intuitionistic fuzzy soft sets are proposed. A prioritization technique for order preference by similarity to the ideal solution based on interval-valued intuitionistic fuzzy soft sets of correlation coefficients and the weighted correlation coefficient is introduced. We also proposed interval-valued intuitionistic fuzzy soft weighted average and interval-valued intuitionistic fuzzy soft weighted geometric operators and developed decision-making techniques based on the proposed operators. By using the developed techniques, a method for solving decision-making problems is proposed. To ensure the applicability of the proposed methods, an illustrative example is given. Finally, we present a comparison of some existing methods with our proposed techniques.
\end{abstract}

\section{Introduction}

Correlation performs a vital part in statistics and engineering; through correlation analysis, the joint relationship of two variables can be used to evaluate the interdependence of two variables. Although probabilistic methods have been applied to various practical engineering problems, there are some obstacles to probabilistic strategies. For example, the probability of a process depends on the large amount of data collected, which is random. However, large-scale complex systems have many fuzzy uncertainties, so it is difficult to obtain accurate probability events. Therefore, the results based on probability theory do not always provide useful information for experts due to the limited quantitative information. Besides, in practical applications, sometimes there is not enough data to properly process standard statistical data. Due to the abovementioned obstacles, the results based on probability theory are not always available to experts. Therefore, probabilistic methods are usually not enough to resolve such inherent uncertainties in the data. A lot of researchers in the world proposed and recommended different approaches to solve those problems that contain uncertainty. First of all, Zadeh developed the notion of fuzzy sets (FSs) [1] to solve those problems which contain uncertainty and vagueness. It is observed that in some cases, circumstances cannot be handled by FSs, and to overcome such types of situations, Turksen [2] gave the idea of intervalvalued fuzzy sets (IVFSs). In some cases, we must deliberate membership unbiased as a nonmembership value for the suitable representation of an object in uncertain and indeterminate conditions that could not be handled by FSs nor by IVFSs. To overcome these difficulties, Atanassov 
presented the idea of intuitionistic fuzzy sets (IFSs) [3]. The theory which was presented by Atanassov only deals with insufficient data because of both the membership and nonmembership values, but the IFSs cannot handle incompatible and imprecise information.

A general mathematical tool was proposed by Molodtsov [4] to deal with indeterminate, fuzzy, and not clearly defined substances known as a soft set (SS). Maji et al. [5] extended the work on SS and developed some operations with their properties. In [6], they also used the SS theory for decision-making. Ali et al. [7] revised the Maji approach to SS and developed some new operations with their properties. De Morgan's law on SS theory was proved [8] by using different operators. Çağman and Enginoğlu [9] developed the concept of soft matrices with operations and discussed their properties, and they also introduced a decision-making method to resolve those problems which contain uncertainty. In [10], they revised the operations proposed by Molodtsov's SS. Maji et al. [11] developed the notion of the fuzzy soft set (FSS) by combining the FS and SS. They also proposed the intuitionistic fuzzy soft sets (IFSSs) with basic operations and properties [12]. Atanassov and Gargov [13] extended the IFS theory and established a new notion which is known as interval-valued intuitionistic fuzzy sets (IFSs). The authors in [14] established a novel technique to solve multiattribute decision-making (MADM) problems by using set pair analysis (SPA) under the IVIFS environment. Yang et al. [15] developed the concept of the interval-valued fuzzy soft set (IVFSS) with operations and proved some important results by combining the IVFS and SS, and they also used the developed notion for decision-making. Jiang et al. [16] proposed the concept of interval-valued intuitionistic fuzzy soft sets (IVIFSSs) by extending the IVIFS, and they also proposed the necessity and possibility of operations on IVIFSS with their properties. In [17], the authors constructed an algorithm based on IVIFSS and used the developed algorithm for decision-making.

Hwang and Yoon [18] developed the TOPSIS method to solve decision-making problems. By using the TOPSIS method, the minimum distance from a positive ideal solution which supports to elect the finest alternative is easily obtained. After the invention of the TOPSIS method, many researchers used the TOPSIS method for decision-making and extended this approach to the fuzzy and intuitionistic fuzzy environment [19-28]. Garg and Kumar [29] developed the idea of linguistic interval-valued Atanassov intuitionistic fuzzy sets and presented basic operational laws, score, and accuracy functions with their properties. Garg and Arora [30] developed a generalized version of the intuitionistic fuzzy soft set (IFSS) with weighted averaging and geometric aggregation operators and constructed a decision-making technique to solve problems under an intuitionistic fuzzy environment. They also extended the Maclaurin symmetric mean (MSM) operators to IFSS based on Archimedean T-conorm and T-norm [31]. The idea of entropy measure and TOPSIS based on the correlation coefficient (CC) has been developed by using complex Q-rung orthopair fuzzy information and used the established techniques for decision-making [32].

In [33], the authors proposed the functional measuring of the interrelation of IFSs, nowadays, known as correlation, and developed its coefficient properties. To measure the interrelation of fuzzy numbers, $\mathrm{Yu}$ [34] established the CC of fuzzy numbers. Evaluating the CC for fuzzy data has been developed by Chiang and Lin [35]. Hung and Wu [36] proposed the centroid method to calculate the CC of IFSs and extended the proposed method to IVIFS. Bustince and Burillo [37] introduced the correlation and CC of IVIFS and proved the decomposition theorem on the correlation of IVIFS. Hong [38] and Mitchell [39] also established the CC for IFSs and IVIFSs, respectively. Garg and Arora introduced the correlation measures on IFSS and constructed the TOPSIS technique based on developed correlation measures [40]. Huang and Guo [41] gave an improved CC on IFS with their properties, and they also established the coefficient of IVIFS. Singh et al. [42] developed the one- and two-parametric generalization of CC on IFS and used the proposed technique in multiattribute group decision-making problems. Thao [43] introduced the variance and covariance to establish the novel CC among IFS. Garg and Arora [44] developed the aggregate operators by using dual hesitant fuzzy soft numbers and utilized the proposed operators to solve MCDM problems. Jana et al. [45] developed various aggregation operators under Q-rung orthopair fuzzy environment.

In this research, the TOPSIS technique extends to IVIFSS information, where the mechanisms are assumed in terms of IVIFSNs. To measure the degree of dependency of IVIFSSs, we propose a new CC on IVIFSSs and examine some properties of developed CC. To achieve the goal accurately, the TOPSIS technique may be extended to solve multiattribute decision-making (MADM) problems. In the present research, our main objective is to introduce a new CC under IVIFSS information and develop the TOPSIS method for IVIFSS based on the proposed CC, intervalvalued intuitionistic fuzzy soft weighted average (IVIFSWA), and interval-valued intuitionistic fuzzy soft weighted geometric (IVIFSWG) operators. To solve MADM problems based on the extended TOPSIS approach, an algorithm is developed and the validity of the proposed technique is checked with a numerical illustration. The correlation measures are given that IVIFSS has been considered for the pairs of IVIFSSs, which will be used to compute the interrelation as well as the scope of dependence between the elements. Since the prevailing IFS and IFSS are special cases of IVIFSSs, therefore, the developed measure is more generalized than the prevailing measures. The CC conserves the linear relationship between the underconsidered elements. To find the closeness coefficient, generally, researchers used the basic TOPSIS method, similarity measures, and distance. Meanwhile, in our developed method, the closeness coefficient can be computed by utilizing the CC.

The rest of the article is organized as follows. In Section 2, we remember some basic definitions such as SS, FSS, IVFSS, IFS, and IVIFSS and IVIFSS with some operations. In Section 3, we propose the correlation and informational energies for IVIFSS and develop the CC and WCC with their properties by using the correlation and informational energies. An extended TOPSIS technique is presented based on 
CC, and an algorithm is developed based on the proposed TOPSIS method to solve the MADM problem with a numerical illustration in Section 4. The IVIFSWA and IVIFSWG operators with decision-making techniques are presented in Section 5. Section 6 provides a comparative analysis of some existing techniques to developed methods. Finally, a comprehensive conclusion and future directions are given in Section 7.

\section{Preliminaries}

In this section, we recollect some basic definitions which will be used in the following sequel, such as SS, FSS, IVFSS, IFS, and IVIFSS.

Definition 1 (see [4]). Let $\mathcal{U}$ be the universal set and $\mathscr{E}$ be the set of attributes concerning $\mathscr{U}$. Let $\mathscr{P}(\mathscr{U})$ be the power set of $\mathcal{U}$ and $\mathscr{A} \subseteq \mathscr{E}$. A pair $(\mathscr{F}, \mathscr{A})$ is called an SS over $\mathcal{U}$, and its mapping is given as

$$
\mathscr{F}: \mathscr{A} \longrightarrow \mathscr{P}(\mathscr{U}) \text {. }
$$

It is also defined as

$$
(\mathscr{F}, \mathscr{A})=\{\mathscr{F}(e) \in \mathscr{P}(\mathcal{U}): e \in \mathscr{E}, \mathscr{F}(e)=\varnothing \text {, if } e \notin \mathscr{A}\} .
$$

Definition 2 (see [11]). Let $F(\mathcal{U})$ be a collection of all fuzzy subsets over $\mathcal{U}$ and $\mathscr{E}$ be a set of attributes. Let $\mathscr{A} \subseteq \mathscr{E}$, and then a pair $(\mathscr{F}, \mathscr{A})$ is called FSS over $\mathcal{U}$, where $\mathscr{F}$ is a mapping such as $\mathscr{F}: \mathscr{A} \longrightarrow F(\mathscr{U})$.

Definition 3 (see [15]). A mapping $\mathscr{F}: \mathscr{A} \longrightarrow F(\mathscr{U})$ is known as an IVFSS and defined as $\mathscr{F}_{\mathcal{U}_{i}}(e)$ $=\left\{\left(u_{i}, \sigma_{\mathscr{A}}\left(u_{i}\right)\right) \mid u_{i} \in \mathscr{U}\right\}$, where $\sigma_{\mathscr{A}}\left(u_{i}\right)$ is the interval-valued fuzzy membership value of $u_{i}$ against parameter $e \in \mathscr{E}, F(\mathcal{U})$ is a collection of interval-valued fuzzy subsets of $\mathcal{U}$, and $\sigma_{\mathscr{A}}\left(u_{i}\right)=\left[\sigma_{\mathscr{A}}^{\ell}\left(u_{i}\right), \sigma_{\mathscr{A}}^{\mathscr{U}}\left(u_{i}\right)\right]$ in which $\sigma_{\mathscr{A}}^{\ell}\left(u_{i}\right)$ and $\sigma_{\mathscr{A}}^{\mathscr{U}}\left(u_{i}\right)$ represent the lower and upper limits of the interval.

Definition 4 (see [3]). An IFS is an object of the form $\mathscr{A}=$ $\left\{\left\langle\left(u_{i}, \sigma_{\mathscr{A}}\left(u_{i}\right), \tau_{\mathscr{A}}\left(u_{i}\right)\right) \mid u_{i} \in \mathscr{U}\right\rangle\right\}$ on a universe $\mathcal{U}$, where $\sigma_{\mathscr{A}}$ and $\tau_{\mathscr{A}}: \longrightarrow[0,1]$ represents the degree of membership and nonmembership, respectively, of any element $u_{i}(\in \mathcal{U})$ to set $\mathscr{A}$ with the following condition $0 \leq \sigma_{\mathscr{A}}\left(u_{i}\right)+\tau_{\mathscr{A}}$ $\left(u_{i}\right) \leq 1$.

Definition 5 (see [12]). A mapping $\mathscr{F}: \mathscr{A} \longrightarrow F(\mathscr{U})$ is known as an IFSS and defined as $\mathscr{F}_{u_{i}}(e)$ $=\left\{\left(u_{i}, \sigma_{\mathscr{A}}\left(u_{i}\right), \tau_{\mathscr{A}}\left(u_{i}\right)\right) \mid u_{i} \in \mathscr{U}\right\}$, where $\sigma_{\mathscr{A}}\left(u_{i}\right)$ and $\tau_{\mathscr{A}}\left(u_{i}\right)$ are the degrees of acceptance and rejection, respectively, for all $u_{i} \in \mathcal{U}$ and $0 \leq \sigma_{\mathscr{A}}\left(u_{i}\right), \tau_{\mathscr{A}}\left(u_{i}\right), \sigma_{\mathscr{A}}\left(u_{i}\right)+\tau_{\mathscr{A}}\left(u_{i}\right) \leq 1$.

Definition 6 (see [13]). An IVIFS is an object of the form $\mathscr{A}=\left\{\left\langle\left(u_{i}, \sigma_{\mathscr{A}}\left(u_{i}\right), \tau_{\mathscr{A}}\left(u_{i}\right)\right) \mid u_{i} \in \mathscr{U}\right\rangle\right\}$ on a universe $\mathcal{U}$, where $\sigma_{\mathscr{A}}$ and $\tau_{\mathscr{A}}: \mathscr{U} \longrightarrow \operatorname{Int}([0,1])$. Int $([0,1])$ represents all closed subintervals of $[0,1]$ which satisfy the following condition $\forall u_{i} \in \mathcal{U}, \sup \sigma_{\mathscr{A}}\left(u_{i}\right)+\sup \tau_{\mathscr{A}}\left(u_{i}\right) \leq 1$. IVIFS ( $\left.\mathcal{U}\right)$ represents the class of all IVIFS over $\mathscr{U}$.

Definition 7 (see [13]). Let $(\mathscr{F}, \mathscr{A})$ and $(\mathscr{g}, \mathfrak{B})$ be two IVIFSs over $\mathcal{U}$, and then

(1) $(\mathscr{F}, \mathscr{A})$ is an interval-valued intuitionistic fuzzy subset of $(q, \mathfrak{B})$; if $\mathscr{A} \subseteq \mathfrak{B}$, then $\mathscr{F}(e) \subseteq \mathscr{G}(e) \forall u_{i} \in \mathscr{U}$ and $e \in \mathscr{A}$, that is, $\sigma_{\mathscr{A}}^{\ell}\left(u_{i}\right) \leq \tau_{\mathfrak{B}}^{\ell}\left(u_{i}\right), \sigma_{\mathscr{A}}^{\mathcal{U}}\left(u_{i}\right) \leq$ $\tau_{\mathfrak{B}}^{\mathcal{U}}\left(u_{i}\right), \sigma_{\mathscr{A}}^{\ell}\left(u_{i}\right) \geq \tau_{\mathfrak{B}}^{\ell}\left(u_{i}\right)$, and $\tau_{\mathscr{A}}^{\mathcal{U}}\left(u_{i}\right) \geq \sigma_{\mathfrak{B}}^{\mathcal{U}}\left(u_{i}\right)$

(2) Let $(\mathscr{F}, \mathscr{A})=\left\{\left\langle\left(u_{i}, \sigma_{\mathscr{A}}\left(u_{i}\right), \tau_{\mathscr{A}}\left(u_{i}\right)\right) \mid u_{i} \in \mathscr{U}\right\rangle\right\}$ be an IVIFS, and then its complement is defined as follows: $(\mathscr{F}, \mathscr{A})^{c}=\left\{\left\langle\left(u_{i}, \tau_{\mathscr{A}}\left(u_{i}\right), \sigma_{\mathscr{A}}\left(u_{i}\right)\right) \mid u_{i} \in \mathscr{U}\right\rangle\right\}$

(3) $(\mathscr{F}, \mathscr{A})=(\mathscr{g}, \mathfrak{B})$, if $(\mathscr{F}, \mathscr{A}) \subseteq(\mathscr{g}, \mathfrak{B})$ and $(\mathscr{g}, \mathfrak{B}) \subseteq$ $(\mathscr{F}, \mathscr{A})$

Definition 8 (see [16]). Let $\mathcal{U}$ and $\mathscr{E}$ be the initial universe and set of parameters, respectively, and IVIFS $(\mathcal{U})$ be the set of all IVIFS of $\mathscr{U}$. Let $\mathscr{A} \subseteq \mathscr{E}$, and then a pair $(\mathscr{F}, \mathscr{A})$ is called IVIFSS over $\mathcal{U}$, where $\mathscr{F}$ is a mapping such that $\mathscr{F}: \mathscr{A} \longrightarrow \operatorname{IVIFS}(\mathcal{U})$.

IVIFSS is a parameterized family of IVIFSs of $\mathcal{U}$, and consequently its universe is $\operatorname{IVIFS}(\mathcal{U})$. There exists a mapping from parameters to IVIFS $(\mathcal{U})$, so we can say that IVIFSS is also a special case of SS.

$\mathscr{F}(e)$ states the interval intuitionistic fuzzy value set of the parameter for any $e \in \mathscr{A}$, and it is an IVIFS of $\mathcal{U}$ where $u_{i} \in \mathcal{U}$ and $e \in \mathscr{A}$ can be expressed in the mathematical form such as $\mathscr{F}(e)=\left\{\left\langle\left(u_{i}, \sigma_{\mathscr{A}}\left(u_{i}\right), \tau_{\mathscr{A}}\left(u_{i}\right)\right) \mid u_{i} \in \mathscr{U}\right\rangle\right\}$. Here, $\sigma_{\mathscr{A}}\left(u_{i}\right)=\left\lceil\sigma_{\mathscr{A}}^{\ell}\left(u_{i}\right), \sigma_{\mathscr{A}}^{\mathscr{U}}\left(u_{i}\right)\right\rceil$ and $\sigma_{\mathscr{A}}(u)=\left[\sigma_{\mathscr{A}}^{\ell}\left(u_{i}\right), \sigma_{\mathscr{A}}^{\mathscr{U}}\left(u_{i}\right)\right]$ represent that the interval-valued fuzzy membership degree of $\mathcal{U}$ is held or not on parameter. Simply, an interval-valued intuitionistic fuzzy soft number (IVIFSN) can be expressed as $\mathscr{F}=\left\langle\left[\sigma^{\ell}, \sigma^{\mathfrak{U}}\right],\left[\tau^{\ell}, \tau^{\mathfrak{U}}\right]\right\rangle$, where $0 \leq \sigma^{\mathcal{U}}+\tau^{\mathscr{U}} \leq 1$.

Definition 9 (see [16]). Let $(\mathscr{F}, \mathscr{A})$ and $(\mathscr{Q}, \mathfrak{B})$ be two IVIFSSs over $\mathcal{U}$, and then

(1) $(\mathscr{F}, \mathscr{A})$ is an interval-valued intuitionistic fuzzy soft subset of $(\mathscr{g}, \mathfrak{B})$; if $\mathscr{A} \subseteq \mathfrak{B}$, and $\forall u_{i} \in \mathcal{U}$ and $e \in \mathscr{A}$, we have $\mathscr{F}(e) \subseteq \mathscr{g}(e)$, that is, $\sigma_{\mathscr{A}}^{\ell}\left(u_{i}\right) \leq \sigma_{\mathfrak{B}}^{\ell}\left(u_{i}\right)$, $\sigma_{\mathscr{A}}^{\mathcal{U}}\left(u_{i}\right) \leq \sigma_{\mathfrak{B}}^{\mathcal{U}}\left(u_{i}\right), \tau_{\mathscr{A}}^{\ell}\left(u_{i}\right) \geq \tau_{\mathfrak{B}}^{\ell}\left(u_{i}\right)$, and $\tau_{\mathscr{A}}^{\mathcal{U}}\left(u_{i}\right) \geq \sigma_{\mathfrak{B}}^{\ell}$ $\left(u_{i}\right)$. It can be represented as $(\mathscr{F}, \mathscr{A}) \subseteq(\mathscr{g}, \mathfrak{B})$.

(2) Let $(\mathscr{F}, \mathscr{A})=\left\{\left\langle\left(u_{i}, \sigma_{\mathscr{A}}\left(u_{i}\right), \tau_{\mathscr{A}}\left(u_{i}\right)\right) \mid u_{i} \in \mathscr{U}\right\rangle\right\}$ be an IVIFSS, and then its complement is defined as follows: $(\mathscr{F}, \mathscr{A})^{c}=\left\{\left\langle\left(u_{i}, \tau_{\mathscr{A}}\left(u_{i}\right), \sigma_{\mathscr{A}}\left(u_{i}\right)\right) \mid u_{i} \in \mathscr{U}\right\rangle\right\}$.

(3) $(\mathscr{F}, \mathscr{A})=(\mathscr{g}, \mathfrak{B})$, if $(\mathscr{F}, \mathscr{A}) \subseteq(\mathscr{g}, \mathfrak{B})$ and $(\mathscr{g}, \mathfrak{B})$ $\subseteq(\mathscr{F}, \mathscr{A})$.

(4) If $\sigma_{\mathscr{A}}\left(u_{i}\right)=[0,0]$, and $\tau_{\mathscr{A}}\left(u_{i}\right)=[1,1] \forall u_{i} \in \mathcal{U}$ and $e \in \mathscr{A}$, then $(\mathscr{F}, \mathrm{A})$ are called null IVIFSS over $\mathcal{U}$. It can be denoted as $\Phi$.

(5) If $\sigma_{\mathscr{A}}\left(u_{i}\right)=[1,1]$, and $\tau_{\mathscr{A}}\left(u_{i}\right)=[0,0] \forall u_{i} \in \mathcal{U}$ and $e \in \mathscr{A}$, then $(\mathscr{F}, \mathrm{A})$ are called absolute IVIFSS over $\mathcal{U}$. It can be denoted as $\Omega$. 


\section{Correlation Coefficient of Interval-Valued Intuitionistic Fuzzy Soft Set}

In this section, the concept of the correlation coefficient and the weighted correlation coefficient of IVIFSS has been proposed with some basic properties.

Definition 10. Let $(\mathscr{F}, \mathscr{A})=\left\{\left\langle\left(u_{i}, \sigma_{\mathscr{A}_{k}}\left(u_{i}\right), \tau_{\mathscr{A}_{k}}\left(u_{i}\right)\right)\right| u_{i}\right.$ $\in \mathscr{U}\rangle\}$ and $(\mathscr{g}, \mathfrak{B})=\left\{\left\langle\left(u_{i}, \sigma_{\mathfrak{B}_{k}}\left(u_{i}\right), \tau_{\mathfrak{B}_{k}}\left(u_{i}\right)\right) \mid u_{i} \in \mathscr{U}\right\rangle\right\}$ be two IVIFSSs defined over a set of attributes $\left\{e_{1}, e_{2}, e_{3}, \ldots, e_{m}\right\}$; here $\sigma_{\mathscr{A}_{k}}^{\ell}\left(u_{i}\right)=\left[\sigma_{\mathscr{A}_{k}}^{\ell}\left(u_{i}\right), \sigma_{\mathscr{A}_{k}}^{\mho}\left(u_{i}\right)\right]$, $\tau_{\mathscr{A}_{k}}^{\ell}\left(u_{i}\right)=\left[\tau_{\mathscr{A}_{k}}^{\ell}\left(u_{i}\right), \tau_{\mathscr{A}_{k}}^{\circlearrowright}\left(u_{i}\right)\right], \quad \sigma_{\mathfrak{B}_{k}}^{\ell}\left(u_{i}\right)=\left[\sigma_{\mathfrak{B}_{k}}^{\ell}\left(u_{i}\right), \sigma_{\mathfrak{B}_{k}}^{\mho^{k}}\left(u_{i}\right)\right]$, and $\tau_{\mathfrak{B}_{k}}^{\ell}\left(u_{i}\right)=\left[\tau_{\mathfrak{B}_{k}}^{\ell}\left(u_{i}\right), \tau_{\mathfrak{B}_{k}}^{\circlearrowright}\left(u_{i}\right)\right]$. Then, the informational intuitionistic energies of $(\mathscr{F}, \mathrm{A})$ and $(\mathscr{g}, \mathfrak{B})$ can be described as follows:

$$
\begin{aligned}
& \varsigma_{\text {IVIFSS }}(\mathscr{F}, \mathscr{A})=\sum_{k=1}^{m} \sum_{i=1}^{n}\left(\left(\sigma_{\mathscr{A}_{k}}^{\ell}\left(u_{i}\right)\right)^{2}+\left(\sigma_{\mathscr{A}_{k}}^{\text {ひ }}\left(u_{i}\right)\right)^{2}+\left(\tau_{\mathscr{A}_{k}}^{\ell}\left(u_{i}\right)\right)^{2}+\left(\tau_{\mathscr{A}_{k}}^{ひ}\left(u_{i}\right)\right)^{2}\right), \\
& \varsigma_{\mathrm{IVIFSS}}(\mathscr{g}, \mathfrak{B})=\sum_{k=1}^{m} \sum_{i=1}^{n}\left(\left(\sigma_{\mathfrak{B}_{k}}^{\ell}\left(u_{i}\right)\right)^{2}+\left(\sigma_{\mathfrak{B}_{k}}^{ひ}\left(u_{i}\right)\right)^{2}+\left(\tau_{\mathfrak{B}_{k}}^{\ell}\left(u_{i}\right)\right)^{2}+\left(\tau_{\mathfrak{B}_{k}}^{ひ}\left(u_{i}\right)\right)^{2}\right) .
\end{aligned}
$$

Definition 11. Let $(\mathscr{F}, \mathscr{A})=\left\{\left\langle\left(u_{i}, \sigma_{\mathscr{A}_{k}}\left(u_{i}\right), \tau_{\mathscr{A}_{k}}\left(u_{i}\right)\right)\right| u_{i}\right.$ $\in \mathscr{U}\rangle\}$ and $(\mathscr{g}, \mathfrak{B})=\left\{\left\langle\left(u_{i}, \sigma_{\mathfrak{B}_{k}}\left(u_{i}\right), \tau_{\mathfrak{B}_{k}}\left(u_{i}\right)\right) \mid u_{i} \in \mathscr{U}\right\rangle\right\}$ be two IVIFSSs, and then the correlation between them is defined as follows:

$$
\varsigma_{\text {IVIFSS }}((\mathscr{F}, \mathscr{A}),(g, \mathfrak{B}))=\sum_{k=1}^{m} \sum_{i=1}^{n}\left(\sigma_{\mathscr{A}_{k}}^{\ell}\left(u_{i}\right) * \sigma_{\mathfrak{B}_{k}}^{\ell}\left(u_{i}\right)+\sigma_{\mathscr{A}_{k}}^{u}\left(u_{i}\right) * \sigma_{\mathfrak{B}_{k}}^{\mathcal{u}}\left(u_{i}\right)+\tau_{\mathscr{A}_{k}}^{\ell}\left(u_{i}\right) * \tau_{\mathfrak{B}_{k}}^{\ell}\left(u_{i}\right)+\tau_{\mathscr{A}_{k}}^{u}\left(u_{i}\right) * \tau_{\mathfrak{B}_{k}}^{u}\left(u_{i}\right)\right) .
$$

Proposition 1. Let $(\mathscr{F}, \mathscr{A})=\left\{\left\langle\left(u_{i}, \sigma_{\mathscr{A}_{k}}\left(u_{i}\right), \tau_{\mathscr{A}_{k}}\left(u_{i}\right)\right)\right| u_{i}\right.$ $\in \mathscr{U}\rangle\}$ and $(\mathscr{g}, \mathfrak{B})=\left\{\left\langle\left(u_{i}, \sigma_{\mathfrak{B}_{k}}\left(u_{i}\right), \tau_{\mathfrak{B}_{k}}\left(u_{i}\right)\right) \mid u_{i} \in \mathscr{U}\right\rangle\right\}$ be two IVIFSSs and $\mathscr{C}_{\text {IVIFSS }}((\mathscr{F}, \mathscr{A}),(\mathscr{q}, \mathfrak{B}))$ be a correlation between them, and then the following properties hold:
(1) $\mathscr{C}_{\text {IVIFSS }}((\mathscr{F}, \mathscr{A}),(\mathscr{F}, \mathscr{A}))=\varsigma_{\text {IVIFSS }}(\mathscr{F}, \mathscr{A})$
(2) $\mathscr{C}_{\text {IVIFSS }}((q, \mathfrak{B}),(\mathfrak{g}, \mathfrak{B}))=\varsigma_{\text {IVIFSS }}(q, \mathfrak{B})$

Proof. The proof is trivial.

Definition 12. Let $(\mathscr{F}, \mathscr{A})=\left\{\left\langle\left(u_{i}, \sigma_{\mathscr{A}_{k}}\left(u_{i}\right), \tau_{\mathscr{A}_{k}}\left(u_{i}\right)\right)\right| u_{i}\right.$ $\in \mathscr{U}\rangle\}$ and $(\mathscr{g}, \mathfrak{B})=\left\{\left\langle\left(u_{i}, \sigma_{\mathfrak{B}_{k}}\left(u_{i}\right), \tau_{\mathfrak{B}_{k}}\left(u_{i}\right)\right) \mid u_{i} \in \mathscr{U}\right\rangle\right\}$ be two IVIFSSs, and then their correlation coefficient is given as $\delta_{\text {IVIFSS }}((\mathscr{F}, \mathscr{A}),(\mathscr{Z}, \mathfrak{B}))$ and expressed as follows:

$$
\delta_{\text {IVIFSS }}((\mathscr{F}, \mathscr{A}),(\mathscr{g}, \mathfrak{B}))=\frac{\mathscr{C}_{\text {IVIFSS }}((\mathscr{F}, \mathscr{A}),(\mathscr{g}, \mathfrak{B}))}{\sqrt{\varsigma_{\text {IVIFSS }}(\mathscr{F}, \mathscr{A})} \sqrt{\varsigma_{\text {IVIFSS }}(\mathscr{g}, \mathfrak{B})}} .
$$

Example 1. Let $(\mathscr{F}, \mathscr{A})$ and $(\mathscr{q}, \mathfrak{B})$ be two IVIFSSs over $\mathscr{U}=$ $\left\{u_{1}, u_{2}\right\}$ and $\mathscr{E}=\{e\}$ be a set of attributes which consist of only one parameter given as follows: $(\mathscr{F}, \mathscr{A})$ $=\left\{\left\langle e,\left(\left(u_{1},[.2, .4],[.4, .5]\right),\left(u_{2},[.3, .5],[.1, .4]\right)\right)\right\rangle\right\} \quad$ and $(\mathfrak{g}, \mathfrak{B})=\left\{\left\langle e,\left(\left(u_{1},[.3, .6],[.1, .4]\right),\left(u_{2},[.3, .5],[.2, .3]\right)\right)\right\rangle\right\}$.

Then, by using Definitions $1-3$, we have $\varsigma_{\text {IVIFSS }}(\mathscr{F}, \mathscr{A})=1.12, \quad S_{\text {IVIFSS }}(\mathscr{Q}, \mathfrak{B})=1.09, \quad$ and $\mathscr{C}_{\text {IVIFSS }}((\mathscr{F}, \mathscr{A}),(\mathscr{g}, \mathfrak{B}))=1.02$. Now, by utilizing equation (6), we get

$$
\delta_{\text {IVIFSS }}((\mathscr{F}, \mathscr{A}),(\mathscr{g}, \mathfrak{B}))=0.92316 .
$$

Proposition 2. Let $(\mathscr{F}, \mathscr{A})=\left\{\left\langle\left(u_{i}, \sigma_{\mathscr{A}_{k}}\left(u_{i}\right), \tau_{\mathscr{A}_{k}}\left(u_{i}\right)\right)\right| u_{i}\right.$ $\in \mathscr{U}\rangle\}$ and $(\mathscr{g}, \mathfrak{B})=\left\{\left\langle\left(u_{i}, \sigma_{\mathfrak{B}_{k}}\left(u_{i}\right), \tau_{\mathfrak{B}_{k}}\left(u_{i}\right)\right) \mid u_{i} \in \mathscr{U}\right\rangle\right\}$ be two IVIFSSs, and then CC between them satisfies the following properties:

(1) $0 \leq \delta_{\text {IVIFSS }}((\mathscr{F}, \mathscr{A}),(\mathscr{q}, \mathfrak{B})) \leq 1$

(2) $\delta_{\text {IVIFSS }}((\mathscr{F}, \mathscr{A}),(\mathscr{q}, \mathfrak{B}))=\delta_{\text {IVIFSS }}((\mathscr{g}, \mathfrak{B}),(\mathscr{F}, \mathscr{A}))$

(3) If $(\mathscr{F}, \mathscr{A})=(\mathscr{g}, \mathfrak{B})$, that is, $\forall i, k, \sigma_{\mathscr{A}_{k}}^{\ell}\left(u_{i}\right)=\sigma_{\mathfrak{B}_{k}}^{\ell}\left(u_{i}\right)$, $\sigma_{\mathscr{A}_{k}}^{ひ}\left(u_{i}\right)=\sigma_{\mathfrak{B}_{k}}^{ひ}\left(u_{i}\right), \tau_{\mathscr{A}_{k}}^{\ell}\left(u_{i}\right)=\tau_{\mathfrak{B}_{k}}^{\ell}\left(u_{i}\right)$, and $\tau_{\mathscr{A}_{k}}^{ひ}\left(u_{i}\right)$ $=\tau_{\mathfrak{B}_{k}}^{\text {ひ }}\left(u_{i}\right)$, then $\delta_{\text {IVIFSS }}((\mathscr{F}, \mathscr{A}),(\mathscr{g}, \mathfrak{B}))=1$

Proof 1. $\delta_{\text {IVIFSS }}((\mathscr{F}, \mathscr{A}),(\mathscr{g}, \mathfrak{B})) \geq 0$ is trivial, and here we only need to prove that $\delta_{\text {IVIFSS }}((\mathscr{F}, \mathscr{A}),(\mathscr{q}, \mathfrak{B})) \leq 1$. 
From equation (5), we have

$$
\begin{aligned}
& \mathscr{C}_{\text {IVIFSS }}((\mathscr{F}, \mathscr{A}),(\mathscr{g}, \mathfrak{B}))=\sum_{k=1}^{m} \sum_{i=1}^{n}\left(\sigma_{\mathscr{A}_{k}}^{\ell}\left(u_{i}\right) * \sigma_{\mathfrak{B}_{k}}^{\ell}\left(u_{i}\right)+\sigma_{\mathscr{A}_{k}}^{ひ}\left(u_{i}\right) * \sigma_{\mathfrak{B}_{k}}^{ひ}\left(u_{i}\right)+\tau_{\mathscr{A}_{k}}^{\ell}\left(u_{i}\right) * \tau_{\mathfrak{B}_{k}}^{\ell}\left(u_{i}\right)+\tau_{\mathscr{A}_{k}}^{ひ}\left(u_{i}\right) * \tau_{\mathfrak{B}_{k}}^{ひ}\left(u_{i}\right)\right) \\
& =\sum_{k=1}^{m}\left(\sigma_{\mathscr{A}_{k}}^{\ell}\left(u_{1}\right) * \sigma_{\mathfrak{B}_{k}}^{\ell}\left(u_{1}\right)+\sigma_{\mathscr{A}_{k}}^{ひ}\left(u_{1}\right) * \sigma_{\mathfrak{B}_{k}}^{ひ}\left(u_{1}\right)+\tau_{\mathscr{A}_{k}}^{\ell}\left(u_{1}\right) * \tau_{\mathfrak{B}_{k}}^{\ell}\left(u_{1}\right)+\tau_{\mathscr{A}_{k}}^{ひ}\left(u_{1}\right) * \tau_{\mathfrak{B}_{k}}^{ひ}\left(u_{1}\right)\right) \\
& +\sum_{k=1}^{m}\left(\sigma_{\mathscr{A}_{k}}^{\ell}\left(u_{2}\right) * \sigma_{\mathfrak{B}_{k}}^{\ell}\left(u_{2}\right)+\sigma_{\mathscr{A}_{k}}^{ひ}\left(u_{2}\right) * \sigma_{\mathfrak{B}_{k}}^{ひ}\left(u_{2}\right)+\tau_{\mathscr{A}_{k}}^{\ell}\left(u_{2}\right) * \tau_{\mathfrak{B}_{k}}^{\ell}\left(u_{2}\right)+\tau_{\mathscr{A}_{k}}^{ひ}\left(u_{2}\right) * \tau_{\mathfrak{B}_{k}}^{ひ}\left(u_{2}\right)\right) \\
& + \\
& \vdots \\
& + \\
& \sum_{k=1}^{m}\left(\sigma_{\mathscr{A}_{k}}^{\ell}\left(u_{n}\right) * \sigma_{\mathfrak{B}_{k}}^{\ell}\left(u_{n}\right)+\sigma_{\mathscr{A}_{k}}^{ひ}\left(u_{n}\right) * \sigma_{\mathfrak{B}_{k}}^{ひ}\left(u_{n}\right)+\tau_{\mathscr{A}_{k}}^{\ell}\left(u_{n}\right) * \tau_{\mathfrak{B}_{k}}^{\ell}\left(u_{n}\right)+\tau_{\mathscr{A}_{k}}^{ひ}\left(u_{n}\right) * \tau_{\mathfrak{B}_{k}}^{ひ}\left(u_{n}\right)\right),
\end{aligned}
$$

$\mathscr{C}_{\text {IVIFSS }}((\mathscr{F}, \mathscr{A}),(\mathscr{g}, \mathfrak{B}))$

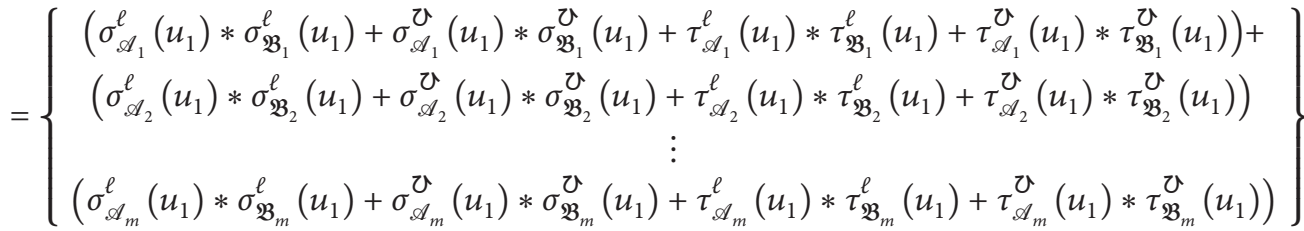

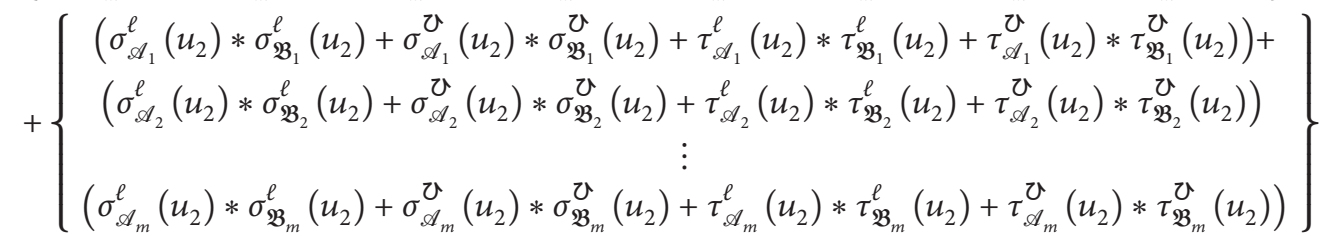

$$
\begin{aligned}
& +
\end{aligned}
$$

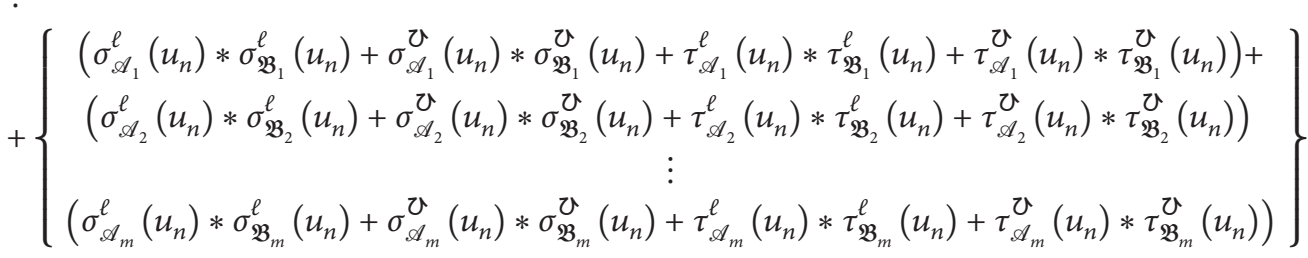

$$
\begin{aligned}
& =\sum_{k=1}^{m}\left(\begin{array}{c}
\left(\sigma_{\mathscr{A}_{k}}^{\ell}\left(u_{1}\right) * \sigma_{\mathfrak{B}_{k}}^{\ell}\left(u_{1}\right)+\sigma_{\mathscr{A}_{k}}^{\circlearrowright}\left(u_{1}\right) * \sigma_{\mathfrak{B}_{k}}^{\circlearrowright}\left(u_{1}\right)\right)+ \\
\left(\sigma_{\mathscr{A}_{k}}^{\ell}\left(u_{2}\right) * \sigma_{\mathfrak{B}_{k}}^{\ell}\left(u_{2}\right)+\sigma_{\mathscr{A}_{k}}^{\circlearrowright}\left(u_{2}\right) * \sigma_{\mathfrak{B}_{k}}^{\mho}\left(u_{2}\right)\right)+\ldots+ \\
\left(\sigma_{\mathscr{A}_{k}}^{\ell}\left(u_{n}\right) * \sigma_{\mathfrak{B}_{k}}^{\ell}\left(u_{n}\right)+\sigma_{\mathscr{A}_{k}}^{\circlearrowright}\left(u_{n}\right) * \sigma_{\mathfrak{B}_{k}}^{\circlearrowright}\left(u_{n}\right)\right)
\end{array}\right) \\
& +\sum_{k=1}^{m}\left(\begin{array}{c}
\left(\tau_{\mathscr{A}_{k}}^{\ell}\left(u_{1}\right) * \tau_{\mathfrak{B}_{k}}^{\ell}\left(u_{1}\right)+\tau_{\mathscr{A}_{k}}^{\circlearrowright}\left(u_{1}\right) * \tau_{\mathfrak{B}_{k}}^{\circlearrowright}\left(u_{1}\right)\right)+ \\
\left(\tau_{\mathscr{A}_{k}}^{\ell}\left(u_{2}\right) * \tau_{\mathfrak{B}_{k}}^{\ell}\left(u_{2}\right)+\tau_{\mathscr{A}_{k}}^{\circlearrowright}\left(u_{2}\right) * \tau_{\mathfrak{B}_{k}}^{\mho}\left(u_{2}\right)\right)+\ldots+ \\
\left(\tau_{\mathscr{A}_{k}}^{\ell}\left(u_{n}\right) * \tau_{\mathfrak{B}_{k}}^{\ell}\left(u_{n}\right)+\tau_{\mathscr{A}_{k}}^{\circlearrowright}\left(u_{n}\right) * \tau_{\mathfrak{B}_{k}}^{\circlearrowright}\left(u_{n}\right)\right)
\end{array}\right) .
\end{aligned}
$$


By using Cauchy-Schwarz inequality,

$$
\begin{aligned}
& \left(\mathscr{C}_{\text {IVIFSS }}(\mathscr{F}, \mathscr{A}),(\mathscr{g}, \mathfrak{B})\right)^{2}
\end{aligned}
$$

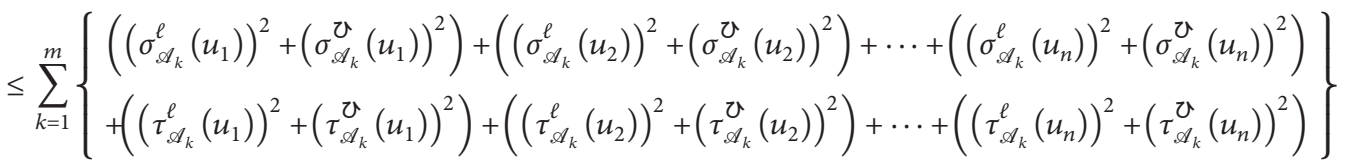

$$
\begin{aligned}
& \times \sum_{k=1}^{m}\left\{\begin{array}{l}
\left(\left(\sigma_{\mathfrak{B}_{k}}^{\ell}\left(u_{1}\right)\right)^{2}+\left(\sigma_{\mathfrak{B}_{k}}^{\text {ひ }}\left(u_{1}\right)\right)^{2}\right)+\left(\left(\sigma_{\mathfrak{B}_{k}}^{\ell}\left(u_{2}\right)\right)^{2}+\left(\sigma_{\mathfrak{B}_{k}}^{ひ}\left(u_{2}\right)\right)^{2}\right)+\cdots+\left(\left(\sigma_{\mathfrak{B}_{k}}^{\ell}\left(u_{n}\right)\right)^{2}+\left(\sigma_{\mathfrak{B}_{k}}^{ひ}\left(u_{n}\right)\right)^{2}\right) \\
+\left(\left(\tau_{\mathfrak{B}_{k}}^{\ell}\left(u_{1}\right)\right)^{2}+\left(\tau_{\mathfrak{B}_{k}}^{ひ}\left(u_{1}\right)\right)^{2}\right)+\left(\left(\tau_{\mathfrak{B}_{k}}^{\ell}\left(u_{2}\right)\right)^{2}+\left(\tau_{\mathfrak{B}_{k}}^{ひ}\left(u_{2}\right)\right)^{2}\right)+\cdots+\left(\left(\tau_{\mathfrak{B}_{k}}^{\ell}\left(u_{n}\right)\right)^{2}+\left(\tau_{\mathfrak{B}_{k}}^{ひ}\left(u_{n}\right)\right)^{2}\right)
\end{array}\right\}, \\
& \left(\varsigma_{\text {IVIFSS }}(\mathscr{F}, \mathscr{A}),(\mathscr{g}, \mathfrak{B})\right)^{2}=\sum_{k=1}^{m} \sum_{i=1}^{n}\left(\left(\sigma_{\mathscr{A}_{k}}^{\ell}\left(u_{i}\right)\right)^{2}+\left(\sigma_{\mathscr{A}_{k}}^{\text {ひ }}\left(u_{i}\right)\right)^{2}+\left(\tau_{\mathscr{A}_{k}}^{\ell}\left(u_{i}\right)\right)^{2}+\left(\tau_{\mathscr{A}_{k}}^{\text {च }}\left(u_{i}\right)\right)^{2}\right) \\
& \times \sum_{k=1}^{m} \sum_{i=1}^{n}\left(\left(\sigma_{\mathfrak{B}_{k}}^{\ell}\left(u_{i}\right)\right)^{2}+\left(\sigma_{\mathfrak{B}_{k}}^{ひ}\left(u_{i}\right)\right)^{2}+\left(\tau_{\mathfrak{B}_{k}}^{\ell}\left(u_{i}\right)\right)^{2}+\left(\tau_{\mathfrak{B}_{k}}^{ひ}\left(u_{i}\right)\right)^{2}\right), \\
& \left(\mathscr{C}_{\text {IVIFSS }}(\mathscr{F}, \mathscr{A}),(\mathscr{g}, \mathfrak{B})\right)^{2} \leq \varsigma_{\text {IVIFSS }}(\mathscr{F}, \mathscr{A}) \times \varsigma_{\text {IVIFSS }}(\mathscr{g}, \mathfrak{B}) .
\end{aligned}
$$

Therefore, $\quad\left(\mathscr{C}_{\text {IVIFSS }}(\mathscr{F}, \mathscr{A}),(\mathscr{g}, \mathfrak{B})\right)^{2} \leq \varsigma_{\text {IVIFSS }}(\mathscr{F}, \mathscr{A})$ $\times \varsigma_{\text {IVIFSS }}(\mathscr{Q}, \mathfrak{B})$. Hence, by using Definition 12 , we get $\varsigma_{\text {IVIFSS }}((\mathscr{F}, \mathscr{A}),(\mathscr{q}, \mathfrak{B})) \leq 1$, so $0 \leq \varsigma_{\text {IVIFSS }}((\mathscr{F}, \mathscr{A}),(\mathscr{q}, \mathfrak{B}))$ $\leq 1$.
Proof 2. The proof is obvious.

Proof 3. From equation (6), we have

$$
\delta_{\text {IVIFSS }}((\mathscr{F}, \mathscr{A}),(q, \mathfrak{B}))=\frac{\sum_{k=1}^{m} \sum_{i=1}^{n}\left(\sigma_{\mathscr{A}_{k}}^{\ell}\left(u_{i}\right) * \sigma_{\mathfrak{B}_{k}}^{\ell}\left(u_{i}\right)+\sigma_{\mathscr{A}_{k}}^{\mathcal{U}}\left(u_{i}\right) * \sigma_{\mathfrak{B}_{k}}^{\mathcal{U}}\left(u_{i}\right)+\tau_{\mathscr{A}_{k}}^{\ell}\left(u_{i}\right) * \tau_{\mathfrak{B}_{k}}^{\ell}\left(u_{i}\right)+\tau_{\mathscr{A}_{k}}^{\mathcal{U}}\left(u_{i}\right) * \tau_{\mathfrak{B}_{k}}^{\mathcal{U}}\left(u_{i}\right)\right)}{\sqrt{\sum_{k=1}^{m} \sum_{i=1}^{n}\left(\left(\sigma_{\mathscr{A}_{k}}^{\ell}\left(u_{i}\right)\right)^{2}+\left(\sigma_{\mathscr{A}_{k}}^{\ell}\left(u_{i}\right)\right)^{2}+\left(\tau_{\mathscr{A}_{k}}^{\ell}\left(u_{i}\right)\right)^{2}+\left(\tau_{\mathscr{A}_{k}}^{\ell}\left(u_{i}\right)\right)^{2}\right)} \sqrt{\sum_{k=1}^{m} \sum_{i=1}^{n}\left(\left(\sigma_{\mathfrak{B}_{k}}^{\ell}\left(u_{i}\right)\right)^{2}+\left(\sigma_{\mathfrak{B}_{k}}^{\ell}\left(u_{i}\right)\right)^{2}+\left(\tau_{\mathfrak{B}_{k}}^{\ell}\left(u_{i}\right)\right)^{2}+\left(\tau_{\mathfrak{B}_{k}}^{\mathcal{U}}\left(u_{i}\right)\right)^{2}\right)}} .
$$

As we know that $\sigma_{\mathscr{A}_{k}}^{\ell}\left(u_{i}\right)=\sigma_{\mathfrak{B}_{k}}^{\ell}\left(u_{i}\right), \sigma_{\mathscr{A}_{k}}^{\text {¿ }}\left(u_{i}\right)=\sigma_{\mathfrak{B}_{k}}^{\text {乙 }}\left(u_{i}\right)$, $\tau_{\mathscr{A}_{k}}^{\ell}\left(u_{i}\right)=\tau_{\mathfrak{B}_{k}}^{\ell}\left(u_{i}\right)$, and $\tau_{\mathscr{A}_{k}}\left(u_{i}\right)=\tau_{\mathfrak{B}_{k}}\left(u_{i}\right) \forall i, k$, we get

$\delta_{\text {IVIFSS }}((\mathscr{F}, \mathscr{A}),(\mathscr{g}, \mathfrak{B}))=\frac{\sum_{k=1}^{m} \sum_{i=1}^{n}\left(\left(\sigma_{\mathfrak{B}_{k}}^{\ell}\left(u_{i}\right)\right)^{2}+\left(\sigma_{\mathfrak{B}_{k}}^{\mho}\left(u_{i}\right)\right)^{2}+\left(\tau_{\mathfrak{B}_{k}}^{\ell}\left(u_{i}\right)\right)^{2}+\left(\tau_{\mathfrak{B}_{k}}^{\mho}\left(u_{i}\right)\right)^{2}\right)}{\sqrt{\sum_{k=1}^{m} \sum_{i=1}^{n}\left(\left(\sigma_{\mathscr{A}_{k}}^{\ell}\left(u_{i}\right)\right)^{2}+\left(\sigma_{\mathscr{A}_{k}}^{ひ}\left(u_{i}\right)\right)^{2}+\left(\tau_{\mathscr{A}_{k}}^{\ell}\left(u_{i}\right)\right)^{2}+\left(\tau_{\mathscr{A}_{k}}^{ひ}\left(u_{i}\right)\right)^{2}\right)} \sqrt{\sum_{k=1}^{m} \sum_{i=1}^{n}\left(\left(\sigma_{\mathfrak{B}_{k}}^{\ell}\left(u_{i}\right)\right)^{2}+\left(\sigma_{\mathfrak{B}_{k}}^{ひ}\left(u_{i}\right)\right)^{2}+\left(\tau_{\mathfrak{B}_{k}}^{\ell}\left(u_{i}\right)\right)^{2}+\left(\tau_{\mathfrak{B}_{k}}^{\mho}\left(u_{i}\right)\right)^{2}\right)}}$, $\delta_{\text {IVIFSS }}((\mathscr{F}, \mathscr{A}),(\mathscr{g}, \mathfrak{B}))=1$.

Thus, proving the required result.

Definition 13. Let $(\mathscr{F}, \mathscr{A})=\left\{\left\langle\left(u_{i}, \sigma_{\mathscr{A}_{k}}\left(u_{i}\right), \tau_{\mathscr{A}_{k}}\left(u_{i}\right)\right)\right| u_{i}\right.$ $\in \mathscr{U}\rangle\}$ and $(\mathscr{g}, \mathfrak{B})=\left\{\left\langle\left(u_{i}, \sigma_{\mathfrak{B}_{k}}\left(u_{i}\right), \tau_{\mathfrak{B}_{k}}\left(u_{i}\right)\right) \mid u_{i} \in \mathscr{U}\right\rangle\right\}$ be two IVIFSSs, and then their correlation coefficient is given as $\delta_{\text {IVIFSS }}^{1}((\mathscr{F}, \mathscr{A}),(\mathscr{Q}, \mathfrak{B}))$ and expressed as follows:

$$
\delta_{\text {IVIFSS }}^{1}((\mathscr{F}, \mathscr{A}),(q, \mathfrak{B}))=\frac{\mathscr{C}_{\text {IVIFSS }}((\mathscr{F}, \mathscr{A}),(\mathscr{q}, \mathfrak{B}))}{\max \left\{\varsigma_{\text {IVIFSS }}(\mathscr{F}, \mathscr{A}), \varsigma_{\text {IVIFSS }}(q, \mathfrak{B})\right\}} .
$$

Proposition 3. Let $(\mathscr{F}, \mathscr{A})=\left\{\left\langle\left(u_{i}, \sigma_{\mathscr{A}_{k}}\left(u_{i}\right), \tau_{\mathscr{A}_{k}}\left(u_{i}\right)\right)\right| u_{i}\right.$ $\in \mathscr{U}\rangle\}$ and $(\mathscr{g}, \mathfrak{B})=\left\{\left\langle\left(u_{i}, \sigma_{\mathfrak{B}_{k}}\left(u_{i}\right), \tau_{\mathfrak{B}_{k}}\left(u_{i}\right)\right) \mid u_{i} \in \mathscr{U}\right\rangle\right\}$ be two IVIFSSs, and then CC between them satisfies the following properties:

(1) $0 \leq \delta_{\text {IVIFSS }}^{1}((\mathscr{F}, \mathscr{A}),(\mathscr{g}, \mathfrak{B})) \leq 1$

(2) $\delta_{\text {IVIFSS }}^{1}((\mathscr{F}, \mathscr{A}),(\mathscr{q}, \mathfrak{B}))=\delta_{\text {IVIFSS }}^{1}((\mathscr{g}, \mathfrak{B}),(\mathscr{F}, \mathscr{A}))$

(3) If $(\mathscr{F}, \mathscr{A})=(\mathscr{g}, \mathfrak{B})$, that is, $\forall i, k, \sigma_{\mathscr{A}_{k}}^{\ell}\left(u_{i}\right)=\sigma_{\mathfrak{B}_{k}}^{\ell}\left(u_{i}\right)$, $\sigma_{\mathscr{A}_{k}}^{ひ}\left(u_{i}\right)=\sigma_{\mathfrak{B}_{k}}^{\mho}\left(u_{i}\right), \quad \tau_{\mathscr{A}_{k}}^{\ell}\left(u_{i}\right)=\tau_{\mathfrak{B}_{k}}^{\ell}\left(u_{i}\right), \quad$ and $\tau_{\mathscr{A}_{k}}^{ひ}\left(u_{i}\right)=\tau_{\mathfrak{B}_{k}}^{ひ}\left(u_{i}\right)$, then $\delta_{\text {IVIFSS }}((\mathscr{F}, \mathscr{A}),(\mathscr{g}, \mathfrak{B}))=1$ 
Proof. The proof is similar to Proposition 2.

Nowadays, considering the weight of IVIFSS is essential for practical applications. The result of a decision may be varying, whenever decision makers adjust the different weights to every element in the universe of discourse. Consequently, it is particularly significant to plan the weight before decision-making. Let $\dot{\omega}_{k}=\left\{\dot{\omega}_{1}, \dot{\omega}_{2}, \dot{\omega}_{3}, \ldots, \dot{\omega}_{m}\right\}$ be a weight vector for experts such as $\dot{\omega}_{k}>0, \sum_{k=1}^{m} \dot{\omega}_{k}=1$, and $\gamma=$ $\left\{\gamma_{1}, \gamma_{2}, \gamma_{3}, \ldots, \gamma_{n}\right\}$ be a weight vector for parameters such as $\gamma_{i}>0, \sum_{i=1}^{n} \gamma_{i}=1$. In the following, we develop the WCC IVIFSS by extending Definitions 12 and 13 .

Definition 14. Let $(\mathscr{F}, \mathscr{A})=\left\{\left\langle\left(u_{i}, \sigma_{\mathscr{A}_{k}}\left(u_{i}\right), \tau_{\mathscr{A}_{k}}\left(u_{i}\right)\right)\right| u_{i}\right.$ $\in \mathscr{U}\rangle\} \quad$ and $(\mathscr{Q}, \mathfrak{B})=\left\{\left\langle\left(u_{i}, \sigma_{\mathfrak{B}_{k}}\left(u_{i}\right), \tau_{\mathfrak{B}_{k}}\left(u_{i}\right)\right) \mid u_{i} \in \mathscr{U}\right\rangle\right\}$ be two IVIFSSs, and then their weighted correlation coefficient is given as $\delta_{\text {WIVIFSS }}((\mathscr{F}, \mathscr{A}),(\mathscr{g}, \mathfrak{B}))$ and expressed as follows:

$$
\delta_{\mathrm{WIVIFSS}}((\mathscr{F}, \mathscr{A}),(\mathscr{g}, \mathfrak{B}))=\frac{\mathscr{C}_{\mathrm{IVIFSS}}((\mathscr{F}, \mathscr{A}),(\mathscr{g}, \mathfrak{B}))}{\max \left\{\varsigma_{\mathrm{IVIFSS}}(\mathscr{F}, \mathscr{A}), \varsigma_{\mathrm{IVIFSS}}(\mathscr{g}, \mathfrak{B})\right\}} .
$$

Definition 15. Let $(\mathscr{F}, \mathscr{A})=\left\{\left\langle\left(u_{i}, \sigma_{\mathscr{A}_{k}}\left(u_{i}\right), \tau_{\mathscr{A}_{k}}\left(u_{i}\right)\right)\right| u_{i}\right.$ $\in \mathscr{U}\rangle\}$ and $(\mathscr{g}, \mathfrak{B})=\left\{\left\langle\left(u_{i}, \sigma_{\mathfrak{B}_{k}}\left(u_{i}\right), \tau_{\mathfrak{B}_{k}}\left(u_{i}\right)\right) \mid u_{i} \in \mathscr{U}\right\rangle\right\}$ be two IVIFSSs, and then their weighted correlation coefficient is also given as $\delta_{\text {WIVIFSS }}^{1}((\mathscr{F}, \mathscr{A}),(\mathscr{g}, \mathfrak{B}))$ and expressed as follows:

$$
\begin{aligned}
& \delta_{\text {WIVIFSS }}^{1}((\mathscr{F}, \mathscr{A}),(\mathscr{g}, \mathfrak{B}))=\frac{\mathscr{C}_{\text {IVIFSS }}((\mathscr{F}, \mathscr{A}),(\mathscr{g}, \mathfrak{B}))}{\max \left\{\varsigma_{\text {IVIFSS }}(\mathscr{F}, \mathscr{A}), \varsigma_{\text {IVIFSS }}(\mathscr{g}, \mathfrak{B})\right\}},
\end{aligned}
$$

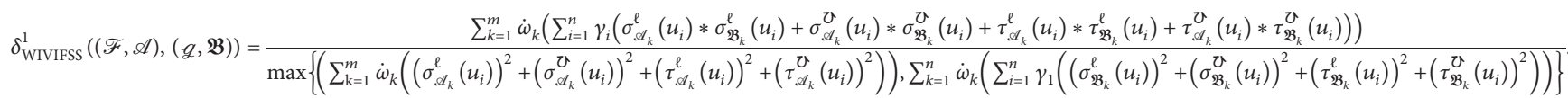

If we consider $\dot{\omega}=\{1 / m, 1 / m, \ldots, 1 / m\}$ and $\gamma=\{1 / n, 1 / n$, $\ldots, 1 / n\}$, then $\delta_{\text {WIVIFSS }}\left(\mathscr{F}_{\breve{A}}, \mathscr{G}_{\breve{B}}\right)$ and $\delta_{\text {WIVIFSS }}^{1}\left(\mathscr{F}_{\breve{A}}, \mathscr{G}_{\breve{B}}\right)$ are reduced to $\delta_{\text {IVIFSS }}\left(\mathscr{F}_{\breve{A}}, \mathscr{G}_{\breve{B}}^{A}\right)$ and $\delta_{\text {IVIFSS }}^{1}\left(\mathscr{F}_{\breve{A}}, \mathscr{G}_{\breve{B}}^{A}\right)$, respectively, defined in Definitions 12 and 13.

Proposition 4. Let $(\mathscr{F}, \mathscr{A})=\left\{\left\langle\left(u_{i}, \sigma_{\mathscr{A}_{k}}\left(u_{i}\right), \tau_{\mathscr{A}_{k}}\left(u_{i}\right)\right)\right| u_{i}\right.$ $\in \mathscr{U}\rangle\}$ and $(\mathscr{G}, \mathfrak{B})=\left\{\left\langle\left(u_{i}, \sigma_{\mathfrak{B}_{k}}\left(u_{i}\right), \tau_{\mathfrak{B}_{k}}\left(u_{i}\right)\right) \mid u_{i} \in \mathcal{U}\right\rangle\right\}$ be two IVIFSSs, and then WCC between them satisfies the following properties:

(1) $0 \leq \delta_{\text {WIVIFSS }}((\mathscr{F}, \mathscr{A}),(\mathscr{G}, \mathfrak{B})) \leq 1$

(2) $\delta_{\text {WIVIFSS }}((\mathscr{F}, \mathscr{A}),(\mathscr{G}, \mathfrak{B}))=\delta_{\text {WIVIFSS }}((\mathscr{G}, \mathfrak{B})$, $(\mathscr{F}, \mathscr{A}))$

(3) If $(\mathscr{F}, \mathscr{A})=(\mathscr{G}, \mathfrak{B})$, that is, $\forall i, k, \sigma_{\mathscr{A}_{k}}^{\ell}\left(u_{i}\right)=\sigma_{\mathfrak{B}_{k}}^{\ell}\left(u_{i}\right)$, $\sigma_{\mathscr{A}_{k}}^{ひ}\left(u_{i}\right)=\sigma_{\mathfrak{B}_{k}}^{ひ}\left(u_{i}\right), \tau_{\mathscr{A}_{k}}^{\ell}\left(u_{i}\right)=\tau_{\mathfrak{B}_{k}}^{\ell}\left(u_{i}\right)$, and $\tau_{\mathscr{A}_{k}}^{\mathcal{A}_{k}}\left(u_{i}\right)$ $=\tau_{\mathfrak{B}_{k}}\left(u_{i}\right)$, then $\delta_{\text {WIVIFSS }}((\mathscr{F}, \mathscr{A}),(\mathscr{G}, \mathfrak{B}))=1$

Proof. Similar to Proposition 2.

\section{TOPSIS Approach on IVIFSS for Solving DM Problems Based on the Correlation Coefficient}

In this section, we develop a technique to solve decisionmaking problems by extending the TOPSIS method for IVIFSS information on the base of the correlation coefficient. Hwang and Yoon [18] developed the TOPSIS method and utilized them to encourage the order of the assessment substances regarding the positive and negative ideal solutions for decision-making matters. By using the TOPSIS method, we can find the best alternative from different alternatives having minimum and maximum distance from
PIS and NIS, respectively. The TOPSIS technique demonstrates that the correlation measure is used to distinguish the positive and negative ideals in the choice ranking. Most researchers used the TOPSIS method to discover the closeness coefficient with a different type of distance and comparability measure. TOPSIS technique with a correlation coefficient is more appropriate to find the closeness coefficient instead of distance and similarity measure, since the correlation measure preserves the linear relationship among those factors which are under consideration. By using the developed CC, an algorithm based on the TOPSIS method will be introduced to select the most appropriate option.

4.1. TOPSIS Method Based on CC for IVIFSS to Solve MADM Problem. Assume a set of " $s$ " alternatives such as $\beta=\left\{\beta^{1}, \beta^{2}, \beta^{3}, \ldots, \beta^{s}\right\}$ for assessment under the team of experts such as $\mathscr{U}=\left\{u_{1}, u_{2}, u_{3}, \ldots, u_{n}\right\}$ with weights $\Omega=\left(\Omega_{1}, \Omega_{1}, \ldots, \Omega_{n}\right)^{T}$ such that $\Omega_{i}>0, \sum_{i=1}^{n} \Omega_{i}=1$. Let $\mathscr{E}=\left\{e_{1}, e_{2}, \ldots, e_{m}\right\}$ be a set of attributes with weights and $\gamma=\left(\gamma_{1}, \gamma_{2}, \gamma_{3}, \ldots, \gamma_{m}\right)^{T}$ be a weight vector for parameters such as $\gamma_{j}>0, \sum_{j=1}^{m} \gamma_{j}=1$. The team of experts $\left\{u_{i}: i=1,2\right.$, $\ldots, n\}$ evaluates the alternatives $\left\{\beta^{(z)}: z=1,2, \ldots, s\right\}$ under the considered parameters $\left\{e_{j}: j=1,2, \ldots, m\right\}$ given in the form of IVIFSNs such as $\mathscr{L}_{i j}^{(z)}=\left(\sigma_{i j}^{(z)}, \tau_{i j}^{(z)}\right)$, where $\sigma_{i j}^{(z)}=\left[\sigma_{i j}^{\ell}, \sigma_{i j}^{\mho}\right]$ and $\tau_{i j}^{(z)}=\left[\tau_{i j}^{\ell}, \tau_{i j}^{\mho}\right]$, in which $0 \leq \sigma_{i j}^{\ell}, \sigma_{i j}^{\mho}$, $\tau_{i j}^{\ell}, \tau_{i j}^{\mho} \leq 1$ and $\sup \sigma_{i j}^{(z)}+\sup \tau_{i j}^{(z)} \leq 1$. So, $\mathscr{L}_{i j}^{(z)}=\left(\left[\sigma_{i j}^{\ell}\right.\right.$, $\left.\left.\sigma_{i j}^{\mho}\right],\left[\tau_{i j}^{\ell}, \tau_{i j}^{ひ}\right]\right)$ for all $i, j$. A flowchart for the method is presented in Figure 1, and stepwise calculated results are presented in Tables 1-8.

Step 1. Construct a matrix for each alternative $\left\{\beta^{(z)}\right.$ : $z=1,2, \ldots, s\}$ as follows: 


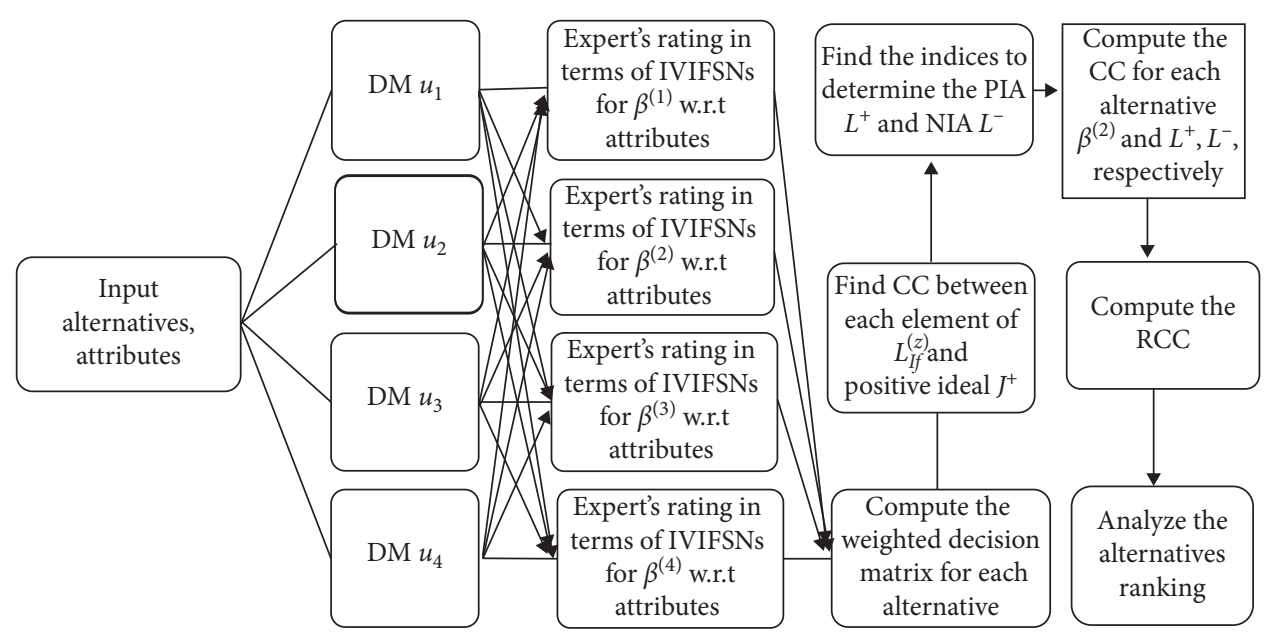

FIGURE 1: Algorithm of the TOPSIS method based on CC for IVIFSS.

TABLE 1: Decision matrix for alternative $\beta^{(1)}$.

\begin{tabular}{|c|c|c|c|c|}
\hline$\beta^{(1)}$ & $e_{1}$ & $e_{2}$ & $e_{3}$ & $e_{4}$ \\
\hline$u_{1}$ & $([.3, .5],[.2, .4])$ & $([.2, .6],[.2, .3])$ & $([.5, .7],[.1, .3])$ & $([.5, .6],[.1, .3])$ \\
\hline$u_{2}$ & $([.5, .7],[.1, .2])$ & $([.4, .6],[.2, .4])$ & $([.3, .4],[.2, .5])$ & $([.6, .8],[.1, .2])$ \\
\hline$u_{3}$ & $([.4, .6],[.2, .3])$ & $([.1, .4],[.2, .5])$ & $([.2, .3],[.1, .6])$ & $([.3, .4],[.2, .5])$ \\
\hline$u_{4}$ & $([.2, .4],[.3, .5])$ & $([.3, .6],[.2, .3])$ & $([.2, .4],[.4, .5])$ & $([.4, .6],[.1, .3])$ \\
\hline
\end{tabular}

TABle 2: Decision matrix for alternative $\beta^{(2)}$.

\begin{tabular}{lcccc}
\hline$\beta^{(2)}$ & $e_{1}$ & $e_{2}$ & $e_{3}$ & $e_{4}$ \\
\hline$u_{1}$ & $([.5, .6],[.2, .3])$ & $([.3, .4],[.4, .6])$ & $([.4, .5],[.3, .5])$ & $([.3, .5],[.4, .5])$ \\
$u_{2}$ & $([.3, .5],[.4, .5])$ & $([.1, .3],[.2, .4])$ & $([.7, .8],[.1, .2])$ & $([.1, .2],[.7, .8])$ \\
$u_{3}$ & $([.6, .7],[.2, .3])$ & $([.3, .4],[.4, .5])$ & $([.5, .8],[.1, .2])$ & $([.1, .2],[.5, .8])$ \\
$u_{4}$ & $([.5, .7],[.1, .2])$ & $([.2, .4],[.5, .6])$ & $([.4, .6],[.2, .3])$ & $([.2, .3],[.4, .6])$ \\
\hline
\end{tabular}

TABLE 3: Decision matrix for alternative $\beta^{(3)}$.

\begin{tabular}{lllll}
\hline$\beta^{(3)}$ & $e_{1}$ & $e_{2}$ & $e_{3}$ \\
\hline$u_{1}$ & $([.4, .5],[.2, .4])$ & $([.3, .5],[.4, .5])$ & $([.4, .6],[.3, .4])$ & $([.3, .4],[.4, .6])$ \\
$u_{2}$ & $([.3, .4],[.4, .6])$ & $([.1, .3],[.3, .7])$ & $([.6, .8],[.1, .2])$ & $([.1, .2],[.6, .8])$ \\
$u_{3}$ & $([.6, .7],[.1, .2])$ & $([.3, .4],[.4, .5])$ & $([.7, .8],[.1, .2])$ & $([.1, .2],[.7, .8])$ \\
$u_{4}$ & $([.5, .6],[.1, .3])$ & $([.2, .3],[.6, .7])$ & $([.4, .6],[.3, .4])$ & $([.3, .4],[.4, .6])$ \\
\hline
\end{tabular}

TABle 4: Decision matrix for alternative $\beta^{(4)}$.

\begin{tabular}{|c|c|c|c|c|}
\hline$\beta^{(4)}$ & $e_{1}$ & $e_{2}$ & $e_{3}$ & $e_{4}$ \\
\hline$u_{1}$ & $([.4, .7],[.1, .2])$ & $([.4, .5],[.2, .4])$ & $([.2, .4],[.3, .4])$ & $([.3, .4],[.2, .4])$ \\
\hline$u_{2}$ & $([.3, .5],[.3, .4])$ & $([.2, .4],[.4, .5])$ & $([.6, .8],[.1, .2])$ & $([.1, .2],[.6, .8])$ \\
\hline$u_{3}$ & $([.6, .7],[.1, .2])$ & $([.4, .5],[.3, .4])$ & $([.5, .7],[.1, .3])$ & $([.1, .3],[.5, .7])$ \\
\hline$u_{4}$ & $([.5, .6],[.1, .3])$ & $([.1, .2],[.7, .8])$ & $([.5, .7],[.2, .3])$ & $([.2, .3],[.5, .7])$ \\
\hline
\end{tabular}

TABLE 5: Weighted decision matrix for $\bar{\beta}^{(1)}$.

\begin{tabular}{ccccc}
\hline $\bar{\beta}^{(1)}$ & $e_{1}$ & $e_{2}$ & $e_{3}$ & $e_{4}$ \\
\hline$u_{1}$ & $([.0307, .0588],[.6687, .9229])$ & $([.0138, .0557],[.9043, .9275])$ & $([.0172, .0297],[.9441, .9703])$ & $([.0507, .0664],[.8414, .9137])$ \\
$u_{2}$ & $([.0474, .0808],[.8511, .8935])$ & $([.0252, .0448],[.9227, .9552])$ & $([.0071, .0102],[.9683, .9862])$ & $([.0535, .0921],[.8709, .9079])$ \\
$u_{3}$ & $([.0265 .0469],[.9189, .9387])$ & $([.0039, .0189],[.9414, .9743])$ & $([.0033, .0053],[.9661, .9924])$ & $([.0159, .0227],[.9301, .9693])$ \\
$u_{4}$ & $([.0308, .0690],[.8449, .9075])$ & $([.0350, .0876],[.8513, .8866])$ & $([.0089, .0202],[.9640, .9727])$ & $([.0595, .1041],[.7586, .8655])$ \\
\hline
\end{tabular}


TABLE 6: Weighted decision matrix for $\bar{\beta}^{(2)}$.

\begin{tabular}{lcccc}
\hline $\bar{\beta}^{(2)}$ & $e_{1}$ & $e_{2}$ & $e_{3}$ & $e_{4}$ \\
\hline$u_{1}$ & $([.0588, .0770],[.8686, .9000])$ & $([.0220, .0314],[.9443, .9686])$ & $([.0127, .0172],[.9703, .9828])$ & $([.0264, .0507],[.9336, .9493])$ \\
$u_{2}$ & $([.0247, .0474],[.9379, .9526])$ & $([.0053, .0177],[.9227, .9552])$ & $([.0238, .0317],[.9549, .9683])$ & $([.0063, .0133],[.9788, .9867])$ \\
$u_{3}$ & $([.0469 .0613],[.9189, .9387])$ & $([.0133, .0189],[.9414, .9559])$ & $([.0103, .0239],[.9661, .9761])$ & $([.0047, .0099],[.9693, .9900])$ \\
$u_{4}$ & $([.0925, .1551],[.7244, .7983])$ & $([.0221, .0498],[.9330, .9502])$ & $([.0202, .0359],[.9377, .9529])$ & $([.0264, .0419],[.8959, .9405])$ \\
\hline
\end{tabular}

Table 7: Weighted decision Matrix for $\bar{\beta}^{(3)}$.

\begin{tabular}{lcccc}
\hline $\bar{\beta}^{(3)}$ & $e_{1}$ & $e_{2}$ & $e_{3}$ & $e_{4}$ \\
\hline$u_{1}$ & $([.0437, .0588],[.8686, .9229])$ & $([.0220, .0424],[.9443, .9576])$ & $([.0127, .0226],[.9703, .9774])$ & $([.0264, .0376],[.9336, .9624])$ \\
$u_{2}$ & $([.0247, .0351],[.9379, .9649])$ & $([.0053, .0177],[.9416, .9823])$ & $([.0182, .0317],[.9549, .9683])$ & $([.0063, .0133],[.9698, .9867])$ \\
$u_{3}$ & $([.0469 .0613],[.8861, .9189])$ & $([.0133, .0189],[.9662, .9743])$ & $([.0179, .0239],[.9661, .9761])$ & $([.0047, .0099],[.9841, .9900])$ \\
$u_{4}$ & $([.0925, .1204],[.7244, .8449])$ & $([.0221, .0350],[.9309, .9649])$ & $([.0202, .0359],[.9529, .0 .9640])$ & $([.0264, .0419],[.8959, .9405])$ \\
\hline
\end{tabular}

TABLE 8: Weighted decision Matrix for $\bar{\beta}^{(4)}$.

\begin{tabular}{ccccc}
\hline $\bar{\beta}^{(4)}$ & $e_{1}$ & $e_{2}$ & $e_{3}$ & $e_{4}$ \\
\hline$u_{1}$ & $([.0437, .0999],[.8175, .8686])$ & $([.0314, .0424],[.9043, .9443])$ & $([.0056, .0127],[.9703, .9774])$ & $([.0264, .0376],[.8863, .9336])$ \\
$u_{2}$ & $([.0247, .0474],[.9192, .9379])$ & $([.0111, .0252],[.9552, .9659])$ & $([.0182, .0317],[.9549, .9683])$ & $([.0063, .0133],[.9698, .9867])$ \\
$u_{3}$ & $([.0469 .0613],[.8861, .9189])$ & $([.0189, .0257],[.9559, .9662])$ & $([.0103, .0179],[.9661, .9821])$ & $([.0047, .0159],[.9693, .9841])$ \\
$u_{4}$ & $([.0925, .1204],[.7244, .8449])$ & $([.0105, .0221],[.9649, .9779])$ & $([.0273, .0470],[.9377, .0 .9529])$ & $([.0264, .0419],[.9202, .9581])$ \\
\hline
\end{tabular}

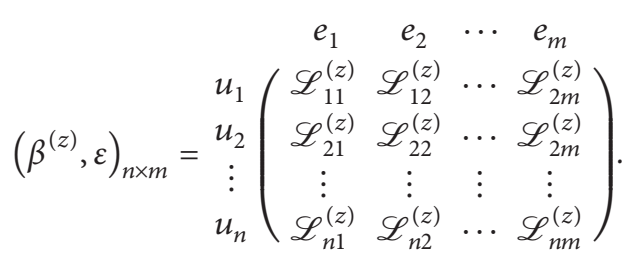

Step 2. Develop the weighted decision matrix $\bar{\beta}^{(z)}=\left(\overline{\mathscr{L}}_{i j}^{(z)}\right)_{n \times m}$, where

$$
\overline{\mathscr{L}}_{i j}^{(z)}=\gamma_{j} \Omega_{i} \mathscr{L}_{i j}^{(z)}=\left(1-\left(\left(1-\left[\sigma_{i j}^{\ell}, \sigma_{i j}^{\circlearrowright}\right]\right)^{\Omega_{i}}\right)^{\gamma_{j}},\left(\left(\left[\tau_{i j}^{\ell}, \tau_{i j}^{\circlearrowright}\right]\right)^{\Omega_{i}}\right)^{\gamma_{j}}\right)=\left(\bar{\sigma}_{i j}^{(z)}, \bar{\tau}_{i j}^{(z)}\right),
$$

in which $\Omega_{i}$ and $\gamma_{j}$ are the weights for the $i^{\text {th }}$ expert and the $j^{\text {th }}$ parameter, respectively.

Step 3. To find the CC of every element of $\overline{\mathscr{L}}_{i j}^{(z)}$ with the perfect positive ideal $\mathscr{I}^{+}=([1,1],[0,0])$ as follows:

$\delta_{\text {IVIFSS }}\left(\overline{\mathscr{L}}_{i j}^{(z)}, \mathscr{I}^{+}\right)=\frac{\mathscr{C}_{\mathrm{IVIFSS}}\left(\overline{\mathscr{L}}_{i j}^{(z)}, \mathscr{I}^{+}\right)}{\sqrt{\varsigma_{\mathrm{IVIFSS}} \overline{\mathscr{L}}_{i j}^{(z)} * \varsigma_{\mathrm{IVIFSS}} \mathscr{I}^{+}}}$.

We obtained a correlation coefficient matrix which can be represented as $\theta^{(z)}=\left(\theta_{i j}^{(z)}\right)_{m \times m}$, where $\theta_{i j}^{(z)}$ is the CC between every element of $\mathscr{L}_{i j}^{(Z)}$ and $\mathscr{I}^{+}$.
Step 4. For each expert $u_{i}$ and parameter from CC matrices $e_{j}$, we find the indices such as $h_{i j}=$ arg $\max _{z}\left\{\theta_{i j}^{(z)}\right\}$ and $g_{i j}=\arg \min _{z}\left\{\theta_{i j}^{(z)}\right\}$ and determine the PIA and NIA based on indices as follows:

$$
\begin{aligned}
& \mathscr{L}^{+}=\left(\sigma^{+}, \tau^{+}\right)_{n \times m}=\left(\bar{\sigma}_{i j}^{\left(h_{i j}\right)}, \bar{\tau}_{i j}^{\left(h_{i j}\right)}\right), \\
& \mathscr{L}^{-}=\left(\sigma^{-}, \tau^{-}\right)_{n \times m}=\left(\bar{\sigma}_{i j}^{\left(g_{i j}\right)}, \bar{\tau}_{i j}^{\left(g_{i j}\right)}\right) .
\end{aligned}
$$

Step 5. To find the CC between each alternative of weighted decision matrices $\bar{\beta}(z)$ and PIA $\mathscr{L}^{+}$as follows:

$$
p^{(z)}=\delta_{\text {IVIFSS }}\left(\bar{\beta}^{(z)}, \mathscr{L}^{+}\right)=\frac{\mathscr{C}_{\text {IVIFSS }}\left(\bar{\beta}^{(z)}, \mathscr{L}^{+}\right)}{\sqrt{\varsigma_{\text {IVIFSS }} \bar{\beta}^{(z)} * \varsigma_{\text {IVIFSS }} \mathscr{L}^{+}}}=\frac{\sum_{j=1}^{m} \sum_{i=1}^{n}\left(\bar{\sigma}_{i j}^{(z)} * \sigma^{+}+\bar{\tau}_{i j}^{(z)} * \tau^{+}\right)}{\sqrt{\sum_{j=1}^{m} \sum_{i=1}^{n}\left(\left({ }^{(z)}\right)_{i j}^{2}+\left({ }^{(z)}\right)^{2}\right)} \sqrt{\sum_{j=1}^{m} \sum_{i=1}^{n}\left(\left(\sigma^{+}\right)^{2}+\left(\tau^{+}\right)^{2}\right)}} .
$$


Step 6. To find the CC between each alternative of weighted decision matrix $\bar{\beta}^{(z)}$ and NIA $\mathscr{L}^{-}$as follows:

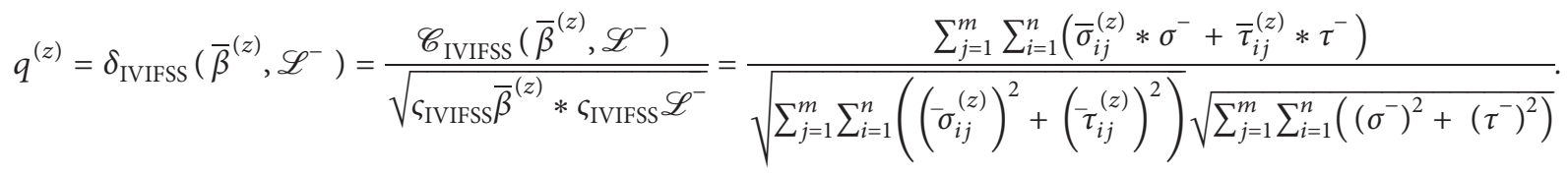

Step 7. To find the closeness coefficient for each alternative:

$$
\mathscr{R}^{(z)}=\frac{\mathscr{K}\left(\bar{\beta}^{(z)}, \mathscr{L}^{-}\right)}{\mathscr{K}\left(\bar{\beta}^{(z)}, \mathscr{L}^{+}\right)+\mathscr{K}\left(\bar{\beta}^{(z)}, \mathscr{L}^{-}\right)},
$$

where $\quad \mathscr{K}\left(\bar{\beta}^{(z)}, \mathscr{L}^{-}\right)=1-q^{(z)}$ and $\mathscr{K}\left(\bar{\beta}^{(z)}, \mathscr{L}^{+}\right)=1-p^{(z)}$.

Step 8. Ranking the alternatives and choosing the best alternative.

4.2. Application of Proposed TOPSIS Technique for Decision-Making. An electrical company calls for the appointment of an electrical engineer, and after initial scrutiny, four candidates (alternatives) remained for further assessment such as $\left\{\beta^{(1)}, \beta^{(2)}, \beta^{(3)}, \beta^{(4)}\right\}$. The managing director of an electrical company hires a team of four decision makers $\left\{u_{1}, u_{2}, u_{3}, u_{4}\right\}$ having weight vectors $(0.25,0.2,0.15,0.4)^{T}$ to conduct the interview. The team of decision makers evaluates the alternatives according to the following parameters: $e_{1}=$ qualification, $e_{2}=$ experience, $e_{3}=$ leadership quality, and $e_{4}=$ personality with weights $(0.35,0.25,0.10,0.30)^{T}$. Every decision maker evaluates the ratings for every alternative in IVIFSN form under the considered parameters. The developed method to find the best alternative for the electric company is given as follows:

Step 1. Develop the decision matrices for each alternative under defined parameters according to the ratings of each decision maker in terms of IVIFSNs.

$$
\begin{array}{r}
\mathscr{L}^{+}=\left[\begin{array}{ccccc}
([.0588, .0770],[.8686, .9000]) & ([.0314, .0424],[.9043, .9443]) & ([.0172, .0297],[.9441, .9703]) & ([.0507, .0664],[.8414, .9137]) \\
([.0247, .0351],[.9379, .9649]) & ([.0053, .0177],[.9416, .9823]) & ([.0071, .0102],[.9683, .9862]) & ([.0063, .0133],[.9698, .9867]) \\
([.0469 .0613],[.8861, .9189]) & ([.0039, .0189],[.9414, .9743]) & ([.0033, .0053],[.9661, .9924]) & ([.0159, .0227],[.9301, .9693]) \\
([.0925, .1204],[.7244, .8449]) & ([.0221, .0350],[.9309, .9649]) & ([.0202, .0359],[.9529, .0 .9640]) & ([.0595, .1041],[.7586, .8655])
\end{array}\right], \\
\mathscr{L}^{-}=\left[\begin{array}{lllll}
([.0437, .0999],[.8175, .8686]) & ([.0220, .0424],[.9443, .9576]) & ([.0056, .0127],[.9703, .9774]) & ([.0264, .0507],[.9336, .9493]) \\
([.0247, .0474],[.9192, .9379]) & ([.0111, .0252],[.9552, .9659]) & ([.0182, .0317],[.9549, .9683]) & ([.0063, .0133],[.9788, .9867]) \\
([.0265 .0469],[.9189, .9387]) & ([.0133, .0189],[.9662, .9743]) & ([.0103, .0239],[.9661, .9761]) & ([.0047, .0099],[.9841, .9900]) \\
([.0925, .1551],[.7244, .7983]) & ([.0105, .0221],[.9649, .9779]) & ([.0202, .0359],[.9377, .9529]) & ([.0264, .0419],[.9202, .9581])
\end{array}\right] .
\end{array}
$$

Step 2. Develop the weighted decision matrices for each alternative by using equation (17).

Step 3. Compute the CC between each alternative $\bar{\beta}^{(z)}$ and perfect positive ideal $\mathscr{I}^{+}=([1,1],[0,0])$ and construct a matrix for each alternative which is known as the CC matrix as follows:

$$
\begin{aligned}
\theta^{(1)} & =\left(\begin{array}{llll}
0.0554 & 0.0379 & 0.0245 & 0.0065 \\
0.0733 & 0.0682 & 0.0089 & 0.0815 \\
0.0395 & 0.0276 & 0.0044 & 0.0203 \\
0.0568 & 0.0703 & 0.0150 & 0.0999
\end{array}\right), \\
\theta^{(2)} & =\left(\begin{array}{llll}
0.0765 & 0.0279 & 0.0153 & 0.0409 \\
0.0381 & 0.0122 & 0.0288 & 0.0099 \\
0.0581 & 0.0169 & 0.0176 & 0.0075 \\
0.1604 & 0.0381 & 0.0297 & 0.0372
\end{array}\right), \\
\theta^{(3)} & =\left(\begin{array}{llll}
0.0571 & 0.0338 & 0.0181 & 0.0337 \\
0.0314 & 0.0119 & 0.0259 & 0.0100 \\
0.0598 & 0.0166 & 0.0215 & 0.0074 \\
0.1340 & 0.0301 & 0.0293 & 0.0372
\end{array}\right), \\
\theta^{(4)}= & \left(\begin{array}{llll}
0.0848 & 0.0399 & 0.0094 & 0.0352 \\
0.0388 & 0.0189 & 0.0259 & 0.0100 \\
0.0598 & 0.0232 & 0.0144 & 0.0105 \\
0.1340 & 0.0168 & 0.0393 & 0.0363
\end{array}\right) .
\end{aligned}
$$

Step 4. Find the PIA and NIA by using equations (19) and (20): 
Step 5. Compute the CC between each alternative of the weighted decision matrix $\bar{\beta}^{(z)}$ and PIA $\mathscr{L}^{+}$.

Correlation coefficient between $\bar{\beta}^{(z)}$ and $\mathscr{L}^{+}$is computed by using equation (21), and $p^{(1)}=0.99701$, $p^{(2)}=0.99822, p^{(3)}=0.99986$, and $p^{(4)}=0.99759$ are obtained.

Step 6. Find the CC between each alternative of the weighted decision matrix $\bar{\beta}^{(z)}$ and NIA $\mathscr{L}^{-}$.

Correlation coefficient between $\bar{\beta}^{(z)}$ and $\mathscr{L}^{-}$is computed by using equation (22), and $q^{(1)}=0.99776$, $q^{(2)}=0.99811, q^{(3)}=0.99988$, and $q^{(4)}=0.99237$ are obtained.

Step 7. By using equation (23), we get the closeness coefficient of alternatives given as follows:

$\mathscr{R}^{(1)}=0.42839, \mathscr{R}^{(2)}=0.51499, \mathscr{R}^{(3)}=0.46154$, and $\mathscr{R}^{(4)}=0.98198$.

Step 8. We conclude from the above calculations that $\mathscr{R}^{(4)}>\mathscr{R}^{(2)}>\mathscr{R}^{(3)}>\mathscr{R}^{(1)}$, so the ranking of the alternatives is described as $\beta^{(4)}>\beta^{(2)}>\beta^{(3)}>\beta^{(1)}$. Therefore, $\beta^{(4)}$ is the best alternative for the electrical company.

\section{Aggregation Operators for Interval-Valued Intuitionistic Fuzzy Soft Numbers (IVIFSNs)}

In this section, interval-valued intuitionistic fuzzy soft weighted average and interval-valued intuitionistic fuzzy soft weighted geometric operators for IVIFSNs are presented.

\subsection{Operational Laws for IVIFSNs}

Definition 16. Let $\aleph_{e}=\left\langle\left[\sigma^{\ell}, \sigma^{\mho}\right],\left[\tau^{\ell}, \tau^{\mho}\right]\right\rangle, \aleph_{e_{11}}=\left\langle\left[\sigma_{11}^{\ell}\right.\right.$, $\left.\left.\sigma_{11}^{ひ}\right],\left[\tau_{11}^{\ell}, \tau_{11}^{ひ}\right]\right\rangle$, and $\aleph_{e_{12}}=\left\langle\left[\sigma_{12}^{\ell}, \sigma_{12}^{ひ}\right],\left[\tau_{12}^{\ell}, \tau_{12}^{ひ}\right]\right\rangle$ be three IVIFSNs and $\alpha$ be a positive real number, and by algebraic norms, we have

(1) $\aleph_{e_{11}} \oplus \aleph_{e_{12}}=\left[\sigma_{11}^{\ell}+\sigma_{12}^{\ell}-\sigma_{11}^{\ell} \sigma_{12}^{\ell}, \sigma_{11}^{ひ}+\sigma_{12}^{ひ}-\sigma_{11}^{ひ} \sigma_{12}^{ひ}\right]$, $\left[\tau_{11}^{\ell} \tau_{12}^{\ell}, \tau_{11}^{\mho} \tau_{12}^{\mho}\right]$

(2) $\begin{aligned} & \aleph_{e_{11}} \otimes \aleph_{e_{12}}=\left[\sigma_{11}^{\ell} \sigma_{12}^{\ell}, \sigma_{11}^{ひ} \sigma_{12}^{ひ}\right],\left[\tau_{11}^{\ell}+\tau_{12}^{\ell}-\tau_{11}^{\ell} \tau_{12}^{\ell},\right. \\ & \left.\tau_{11}^{\mho_{11}}+\tau_{12}^{\sigma^{2}}-\tau_{11}^{\circlearrowright} \tau_{12}^{\circlearrowright}\right]\end{aligned}$

(3) $\alpha \aleph_{e}=\left\langle\left[1-\left(1-\left[\sigma^{\ell}, \sigma^{ひ}\right]\right)^{\alpha},\left(\left[\tau^{\ell}, \tau^{ひ}\right]\right)^{\alpha}\right]\right\rangle$

(4) $\aleph_{e}^{\alpha}=\left\langle\left[\left(\left[\sigma^{\ell}, \sigma^{\mho}\right]\right)^{\alpha}, 1-\left(1-\left[\sigma^{\ell}, \sigma^{\mho}\right]\right)^{\alpha}\right]\right\rangle$

Some averaging and geometric aggregation operators for IVIFSNs have been defined based on the above laws for the collection of IVIFSN $\Delta$.

Definition 17. Let $\aleph_{e_{i j}}=\left\langle\left[\sigma_{i j}^{\ell}, \sigma_{i j}^{ひ}\right],\left[\tau_{i j}^{\ell}, \tau_{i j}^{\circlearrowright}\right]\right\rangle$ be a collection of IVIFSNs, and $\Omega_{i}$ and $\gamma_{j}$ are the weight vector for experts and parameters, respectively, with given conditions $\Omega_{i}>0, \quad \sum_{i=1}^{n} \Omega_{i}=1 ; \quad \gamma_{j}>0, \quad \sum_{j=1}^{m} \gamma_{j}=1, \quad$ where $(i=1,2, \ldots, n$ and $j=1,2, \ldots, m)$. Then, the IVIFSWA operator is defined as IVIFSWA: $\Delta^{n} \longrightarrow \Delta$ defined as follows:

$$
\operatorname{IVIFSWA}\left(\aleph_{e_{11}}, \aleph_{e_{12}}, \ldots, \aleph_{e_{n m}}\right)=\oplus_{j=1}^{m} \gamma_{j}\left(\oplus_{i=1}^{n} \Omega_{i} \aleph_{e_{i j}}\right)
$$

Theorem 1. Let $\aleph_{e_{i j}}=\left\langle\left[\sigma_{i j}^{\ell}, \sigma_{i j}^{\mho}\right],\left[\tau_{i j}^{\ell}, \tau_{i j}^{\mho}\right]\right\rangle$ be an IVIFSN, where $(i=1,2, \ldots, n$ and $j=1,2, \ldots, m)$, and the aggregated value is also an IVIFSN, such as

$$
\operatorname{IVIFSWA}\left(\aleph_{e_{11}}, \aleph_{e_{12}}, \ldots, \aleph_{e_{n m}}\right)=\left\langle 1-\prod_{j=1}^{m}\left(\prod_{i=1}^{n}\left(1-\left[\sigma_{i j}^{\ell}, \sigma_{i j}^{\mho}\right]\right)^{\Omega_{i}}\right)^{\gamma_{j}}, \prod_{j=1}^{m}\left(\prod_{i=1}^{n}\left(\left[\tau_{i j}^{\ell}, \tau_{i j}^{ひ}\right]\right)^{\Omega_{i}}\right)^{\gamma_{j}}\right\rangle .
$$

Proof. Similar to Theorem 3.1 [46].

Example 2. Let $\mathcal{U}=\left\{u_{1}, u_{2}, u_{3}, u_{4}\right\}$ be a set of decision makers, who are going to describe the beauty of a city under the defined set of attributes $\mathscr{E}=\left\{e_{1}, e_{2}, e_{3}\right\}$. Assume $\Omega_{i}=(0.35,0.25,0.10,0.30)^{T}$ and $\gamma_{j}=(0.3,0.5,0.2)^{T}$ be the weight vectors for experts and attributes, respectively. The assumed rating values of the experts for each attribute in the form of IVIFSNs $(\aleph, \mathscr{E})=\left\langle\left[\sigma_{i j}^{\ell}, \sigma_{i j}^{ひ}\right],\left[\tau_{i j}^{\ell}, \tau_{i j}^{ひ}\right]\right\rangle_{4 \times 3}$ are given as follows:

$$
(\aleph, \mathscr{E})=\left[\begin{array}{ccc}
([.3, .5],[.2, .4]) & ([.2, .6],[.2, .3]) & ([.5, .7],[.1, .3]) \\
([.5, .7],[.1, .2]) & ([.4, .6],[.2, .4]) & ([.3, .4],[.2, .5]) \\
([.4 .6],[.2, .3]) & ([.1, .4],[.2, .5]) & ([.2, .3],[.1, .6]) \\
([.2, .4],[.3, .5]) & ([.3, .6],[.2, .3]) & ([.2, .4],[.4, .5])
\end{array}\right] .
$$


By using equation (27),

$$
\begin{aligned}
& \operatorname{IVIFSWA}\left(\aleph_{e_{11}}, \aleph_{e_{12}}, \ldots, \aleph_{e_{43}}\right)=\left\langle 1-\prod_{j=1}^{3}\left(\prod_{i=1}^{4}\left(1-\left[\sigma_{i j}^{\ell}, \sigma_{i j}^{\mho}\right]\right)^{\Omega_{i}}\right)^{\gamma_{j}}, \prod_{j=1}^{3}\left(\prod_{i=1}^{4}\left(\left[\tau_{i j}^{\ell}, \tau_{i j}^{\text {ひ }}\right]\right)^{\Omega_{i}}\right)^{\gamma_{j}}\right\rangle \\
& =1-\left\langle\left(\begin{array}{c}
\left\{[.5, .7]^{0.35}[.3, .5]^{0.25}[.4, .6]^{0.1}[.6, .8]^{0.3}\right\}^{.3}\left\{[.4, .8]^{0.35}[.4, .6]^{0.25}[.6, .9]^{0.1}[.4, .7]^{0.3}\right\} \\
\left\{[.3, .5]^{0.35}[.6, .7]^{0.25}[.7, .8]^{0.1}[.6, .8]^{0.3}\right\}^{.2}
\end{array}\right)\right. \\
& \left.\left(\begin{array}{c}
\left\{[.2, .4]^{0.35}[.1, .2]^{0.25}[.2, .3]^{0.1}[.3, .5]^{0.3}\right\}^{.3}\left\{[.2, .3]^{0.35}[.2, .4]^{0.25}[.2, .5]^{0.1}[.2, .3]^{0.3}\right\} \\
\left\{[.1, .3]^{0.35}[.2, .5]^{0.25}[.1, .6]^{0.1}[.4, .5]^{0.3}\right\}^{.2}
\end{array}\right)\right) \\
& =\langle[0.3098,0.5554],[0.1929,0.3582]\rangle \text {. }
\end{aligned}
$$

Definition 18. Let $\aleph_{e_{i j}}=\left\langle\left[\sigma_{i j}^{\ell}, \sigma_{i j}^{ひ}\right],\left[\tau_{i j}^{\ell}, \tau_{i j}^{ひ}\right]\right\rangle$ be an IVIFSN, and the experts $u_{\mathrm{i}}$ 's and parameters $e_{j}$ 's have standardized weight vectors $\Omega_{i}$ and $\gamma_{j}$, respectively; then the IVIFSWG operator is defined as

$$
\operatorname{IVIFSWG}\left(\aleph_{e_{11}}, \aleph_{e_{12}}, \ldots, \aleph_{e_{n m}}\right)=\otimes_{j=1}^{m}\left(\otimes_{i=1}^{n} \aleph_{e_{n m}}^{\Omega_{i}}\right)^{\gamma_{j}} .
$$

Theorem 2. By using the IVIFSWG operator, the obtained value is also an IVIFSN and given as follows:

$$
\begin{aligned}
& \operatorname{IVIFSWA}\left(\aleph_{e_{11}}, \aleph_{e_{12}}, \ldots, \aleph_{e_{n m}}\right) \\
& =\left\langle\prod_{j=1}^{m}\left(\prod_{i=1}^{n}\left(\left[\sigma_{i j}^{\ell}, \sigma_{i j}^{\mho}\right]\right)^{\Omega_{i}}\right)^{\gamma_{j}}, 1-\prod_{j=1}^{m}\left(\prod_{i=1}^{n}\left(1-\left[\tau_{i j}^{\ell}, \tau_{i j}^{\mho}\right]\right)^{\Omega_{i}}\right)^{\gamma_{j}}\right\rangle .
\end{aligned}
$$

Proof. Similar to Theorem 3.2 [46].

Example 3. Let $\mathcal{U}=\left\{u_{1}, u_{2}, u_{3}, u_{4}\right\}$ be a set of experts with weight vector $\Omega_{i}=(0.35,0.25,0.10,0.30)^{T}$, and all the experts give their suggestion for the beauty of a city under considered attributes $\mathscr{E}=\left\{e_{1}, e_{2}, e_{3}\right\}$ with weight vector $\gamma_{j}=(0.3,0.5,0.2)^{T}$. Suggested values of the experts in the form of IVIFSNs are given $(\aleph, \mathscr{E})$ in the form of a decision matrix as follows:

$$
(\aleph, \mathscr{E})=\left[\begin{array}{cccc}
([.3, .5],[.2, .4]) & ([.2, .6],[.2, .3]) & ([.5, .7],[.1, .3]) \\
([.5, .7],[.1, .2]) & ([.4, .6],[.2, .4]) & ([.3, .4],[.2, .5]) \\
([.46],[.2, .3]) & ([.1, .4],[.2, .5]) & ([.2, .3],[.1, .6]) \\
([.2, .4],[.3, .5]) & ([.3, .6],[.2, .3]) & ([.2, .4],[.4, .5])
\end{array}\right] .
$$

By using equation (31), we get

$\operatorname{IVIFSWG}\left(\aleph_{e_{11}}, \aleph_{e_{12}}, \ldots, \aleph_{e_{43}}\right)=\langle[.2779, .5364],[.2079, .3797]\rangle$.
5.2. Decision-Making Approach with the Proposed Operators. An MADM approach is presented here based on the proposed operators and describes numerical examples for showing their effectivity. 
5.2.1. Proposed Approach. Assume a set of " $s$ " alternatives such as $\beta=\left\{\beta^{1}, \beta^{2}, \beta^{3}, \ldots, \beta^{s}\right\}$ for assessment under the team of experts such as $\mathscr{U}=\left\{u_{1}, u_{2}, u_{3}, \ldots, u_{n}\right\}$ with weights $\Omega=\left(\Omega_{1}, \Omega_{1}, \ldots, \Omega_{n}\right)^{T}$ such that $\Omega_{i}>0, \sum_{i=1}^{n} \Omega_{i}=1$. Let $\mathscr{E}=\left\{e_{1}, e_{2}, \ldots, e_{m}\right\}$ be a set of attributes with weights and $\gamma=\left(\gamma_{1}, \gamma_{2}, \gamma_{3}, \ldots, \gamma_{m}\right)^{T}$ be a weight vector for parameters such as $\gamma_{j}>0, \sum_{j=1}^{m} \gamma_{j}=1$. The team of experts $\left\{u_{i}: i=1,2\right.$, $\ldots, n\}$ evaluates the alternatives $\left\{\beta^{(z)}: z=1,2, \ldots, s\right\}$ under the considered parameters $\left\{e_{j}: j=1,2, \ldots, m\right\}$ given in the form of IVIFSNs such as $\mathscr{L}_{i j}^{(z)}=\left(\sigma_{i j}^{(z)}, \tau_{i j}^{(z)}\right)$, where $\sigma_{i j}^{(z)}=\left[\sigma_{i j}^{\ell}, \sigma_{i j}^{\mho}\right]$ and $\tau_{i j}^{(z)}=\left[\tau_{i j}^{\ell}, \tau_{i j}^{\circlearrowright}\right]$, in which $0 \leq \sigma_{i j}^{\ell}, \sigma_{i j}^{\mho}, \tau_{i j}^{\ell}, \tau_{i j}^{\mho} \leq 1$ and $\sup \sigma_{i j}^{(z)}+\sup \tau_{i j}^{(z)} \leq 1$. So, $\mathscr{L}_{i j}^{(z)}=\left(\left[\sigma_{i j}^{\ell}, \sigma_{i j}^{\circlearrowright}\right],\left[\tau_{i j}^{\ell}, \tau_{i j}^{\circlearrowright}\right]\right)$ for all $i, j$. Experts give their preferences for each alternative in terms of IVIFSNs such as $\aleph_{e_{i j}}=\left\langle\left[\sigma_{i j}^{\ell}, \sigma_{i j}^{\mho}\right],\left[\tau_{i j}^{\ell}, \tau_{i j}^{\circlearrowright}\right]\right\rangle$ and are given in Tables 1-4. Develop the aggregated IVIFSNs $\Delta_{k}$ based on the expert's preference values for each alternative by using IVIFSWG and IVIFSWA operators. Finally, utilizing the alternative, the scoring values [47] of the aggregated IVIFSNs are obtained for the ranking of the alternatives. The algorithm is presented in Figure 2.

\subsubsection{Algorithm}

Step 1. Develop the interval-valued intuitionistic fuzzy soft matrix for each alternative.

Step 2. Aggregate the IVIFSNs for each alternative into a collective decision matrix $\Delta_{k}$ by using the IVIFSWA or IVIFSWG operator.

Step 3. Find the scoring values $\Delta_{k}$ for each alternative [47]:

$$
S\left(\Delta_{k}\right)=\frac{\sigma_{i j}^{\ell}+\sigma_{i j}^{ひ}+\tau_{i j}^{\ell}+\tau_{i j}^{\circlearrowright}}{4}
$$

Step 4. Analyze the ranking.

5.2.3. Numerical Example. An electrical organization calls for the appointment of an electrical engineer. After the first preliminary review, there are still four candidates (substitutes) that need further evaluation, such as $\left\{\beta^{(1)}, \beta^{(2)}, \beta^{(3)}\right.$, $\left.\beta^{(4)}\right\}$. The manager of the company hires a group of four decision makers $\left\{u_{1}, u_{2}, u_{3}, u_{4}\right\}$ whose weight vector is $(0.25,0.2,0.15,0.4)^{T}$. The decision makers give their opinion for each alternative under considered parameters $\left\{e_{1}, e_{2}, e_{3}\right.$, $\left.e_{4}\right\}$ with weights $(0.35,0.25,0.10,0.30)^{T}$. Listed below are the steps to get the best alternative by utilizing the developed method.

\subsubsection{By Using the IVIFSWA Operator}

Step 1. These experts will evaluate the condition in the case of IVIFSNs, and there are just four alternatives;

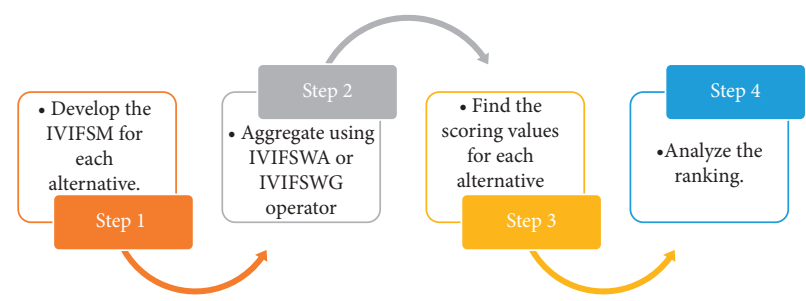

FIgURE 2: Decision-making approaches with the proposed operators.

parameters and a summary of their scores are given in Tables 1-4.

Step 2. The opinions of the experts for each alternative are aggregated by using equation (27), and hence we get

$\Delta_{1}=\langle[.3547 .5695],[.1689, .3457]\rangle$,

$\Delta_{2}=\langle[.3303 .4884],[.3018, .4429]\rangle$,

$\Delta_{3}=\langle[.4296 .5670],[.2988, .4815]\rangle$,

and

$\Delta_{4}=\langle[.3391 .4956],[.2473, .7105]\rangle$.

Step 3. Scoring values by using equation (34):

$S\left(\Delta_{1}\right)=0.3597, \quad S\left(\Delta_{2}\right)=0.3909, \quad S\left(\Delta_{3}\right)=0.4442$, and $S\left(\Delta_{4}\right)=0.4631$.

Step 4. Therefore, the ranking of the alternatives is as follows $\quad S\left(\Delta_{4}\right)>S\left(\Delta_{3}\right)>S\left(\Delta_{2}\right)>S\left(\Delta_{1}\right) . \quad$ So, $\beta^{(4)}>\beta^{(3)}>\beta^{(2)}>\beta^{(1)}$, and hence the alternative $\beta^{(4)}$ is the most suitable alternative for the company.

\subsubsection{By Using the IVIFSWG Operator}

Step 1. The experts will evaluate the condition in the case of IVIFSNs, and there are just four alternatives; parameters and a summary of their scores are given in Tables 1-4.

Step 2. Experts' opinions on each alternative are summarized by using equation (30). Therefore, we get

$\Delta_{1}=\langle[.3144 .5379],[.1819, .3711]\rangle$,

$\Delta_{2}=\langle[.2815 .4420],[.3546, .5037]\rangle$,

$\Delta_{3}=\langle[.2904 .4223],[.3761, .5547]\rangle$,

and

$\Delta_{4}=\langle[.2713 .5445][.3530, .5200]\rangle$.

Step 3. Scoring values by using equation (34):

$S\left(\Delta_{1}\right)=0.3533, \quad S\left(\Delta_{2}\right)=0.3955, \quad S\left(\Delta_{3}\right)=0.4109$, and $S\left(\Delta_{4}\right)=0.4222$.

Step 4. Therefore, the ranking of the alternatives is as follows $\quad S\left(\Delta_{4}\right)>S\left(\Delta_{3}\right)>S\left(\Delta_{2}\right)>S\left(\Delta_{1}\right) . \quad$ So, $\beta^{(4)}>\beta^{(3)}>\beta^{(2)}>\beta^{(1)}$, and hence the alternative $\beta^{(4)}$ is the most suitable alternative for the company.

\section{Result Comparison and Discussion}

Since interval-valued soft sets are more precise than fuzzy soft sets when the case study consists of uncertain and inconsistent information, thus, its extension, an interval-valued intuitionistic fuzzy soft set, will play a vital role in uncertain and indeterminate problems. Existing 


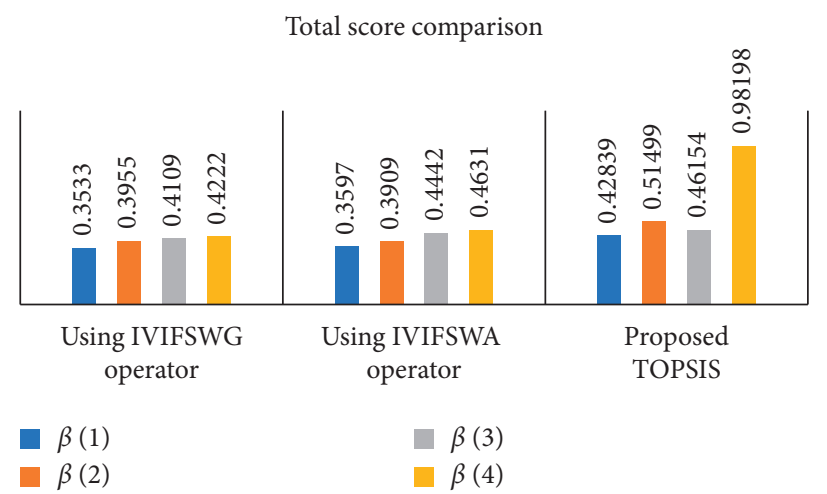

FIgURE 3: Comparison of the alternative rank with the proposed algorithm.

TABLE 9: Alternative rank comparison using the existing and proposed techniques.

\begin{tabular}{lcc}
\hline Method & Alternative final ranking & Optimal choice \\
\hline Proposed algorithm using IVIFSWG operator & $\beta^{(4)}>\beta^{(3)}>\beta^{(2)}>\beta^{(1)}$ & $\beta^{(4)}$ \\
Proposed algorithm using IVIFSWA operator & $\beta^{(4)}>\beta^{(3)}>\beta^{(2)}>\beta^{(1)}$ & $\beta^{(4)}$ \\
Proposed TOPSIS algorithm & $\beta^{(4)}>\beta^{(2)}>\beta^{(3)}>\beta^{(1)}$ & $\beta^{(4)}$ \\
Mukherjee and Sarkar [50] & $\beta^{(4)}>\beta^{(2)}>\beta^{(3)}>\beta^{(1)}$ & $\beta^{(4)}$ \\
Imtiaz et al. [48] & $\beta^{(4)}>\beta^{(3)}>\beta^{(2)}>\beta^{(1)}$ & $\beta^{(4)}$ \\
Wu and Su [49] & $\beta^{(4)}>\beta^{(3)}>\beta^{(2)}>\beta^{(1)}$ & $\beta^{(4)}$ \\
\hline
\end{tabular}

TABLE 10: Alternative final scores using the proposed techniques.

\begin{tabular}{lccc}
\hline Alternative & Proposed algorithm using IVIFSWG operator & Proposed algorithm using IVIFSWA operator & Proposed TOPSIS algorithm \\
\hline$\beta^{(1)}$ & 0.3533 & 0.3597 & 0.42839 \\
$\beta^{(2)}$ & 0.3955 & 0.3909 & 0.51499 \\
$\beta^{(3)}$ & 0.4109 & 0.4442 & 0.46154 \\
$\beta^{(4)}$ & 0.4222 & 0.4631 & 0.98198 \\
\hline
\end{tabular}

methodologies of IFSSs have some limitations on membership and nonmembership grades, and they cannot deal with parameterizations. The proposed algorithms of IVFSSs enhance the existing methodologies, and the decision maker can choose the values from the interval with membership and nonmembership as its limitation. There is a strong relationship between the proposed model and MADM problems. In this paper, we have proposed two types of algorithms. Firstly, the TOPSIS algorithm is proposed based on the correlation coefficients, and a numerical example is solved. Secondly, averaging aggregate and geometric aggregate operators is proposed. Moving on to the next, all the algorithms are applied to the real-world problem, i.e., the selection of electrical engineers. The graphical representation of all results is presented in Figure 3. The results show that the proposed algorithms are valid and practical. Finally, the rank of all the alternatives using the existing algorithms gives the same final decision that $\beta^{(4)}$ is selected for the post of the "electrical engineer." All rankings are also calculated by applying the existing approaches. The proposed methods are also compared with other existing methods by Imtiaz et al. [48], Wu and Su [49], and Mukherjee and Sarkar [50]. The listing in Table 9 shows the results of the comparison in the final ranking of the top 4 alternatives. It can be observed that the best selections made by the proposed methods are compared with the already established methods which are expressive in itself and approves the reliability and validity of the proposed method. The final score values of the proposed TOPSIS method, IVIFSWA, and IVIFSWG can be seen in Table 10.

\section{Conclusion}

The investigated study utilizes the IVIFSS to address the unsatisfactory, obscure, and inconsistent data by considering the membership degree and nonmembership degree over the set of parameters. The novel concept of the correlation coefficient and weighted correlation coefficient for IVIFSS with their properties is proposed in the present research. Based on the developed correlation measures, an extended TOPSIS method has been introduced by considering the set of attributes and decision makers. The proposed method not only debates the discrimination but also handles the degree of similarity to prevent short decisions during observation. We also develop the correlation matrices and find the correlation indices; the PIA and NIA are also developed by using the correlation indices. To find the ranking of the alternatives, we define the closeness coefficient for the developed method. Moreover, the IVIFSWA and IVIFSWG operators have also defined and presented the decisionmaking techniques based on developed operators. Finally, a numerical illustration has been described to solve the 
MADM problem by using the proposed TOPSIS method and other developed techniques. Furthermore, we have compared our proposed method with some already existing techniques. In the future, we plan to extend our work to (i) CC for multipolar interval-valued intuitionistic fuzzy soft sets, (ii) CC for interval-valued neutrosophic soft sets, and (iii) CC for the multipolar interval-valued neutrosophic soft sets.

\section{Data Availability}

No such type of data are used in this manuscript.

\section{Conflicts of Interest}

The authors declare that they have no conflicts of interest.

\section{Acknowledgments}

This research was partially supported by a grant of the National Natural Science Foundation of China (11971384).

\section{References}

[1] L. A. Zadeh, "Fuzzy sets," Information and Control, vol. 8, no. 3, pp. 338-353, 1965.

[2] I. B. Turksen, "Interval valued fuzzy sets based on normal forms," Fuzzy Sets and Systems, vol. 20, no. 2, pp. 191-210, 1986.

[3] K. Atanassov, "Intuitionistic fuzzy sets," Fuzzy Sets and Systems, vol. 20, pp. 87-96, 1986.

[4] D. Molodtsov, "Soft set theory first results," Computers \& Mathematics with Applications, vol. 37, pp. 19-31, 1999.

[5] P. K. Maji, R. Biswas, and A. R. Roy, "Soft set theory," Computers \& Mathematics with Applications, vol. 45, no. 4-5, pp. 555-562, 2003.

[6] P. K. Maji, A. R. Roy, and R. Biswas, "An application of soft sets in a decision making problem," Computers \& Mathematics with Applications, vol. 44, no. 8-9, pp. 1077-1083, 2002.

[7] M. I. Ali, F. Feng, X. Liu, W. K. Min, and M. Shabir, "On some new operations in soft set theory," Computers \& Mathematics with Applications, vol. 57, no. 9, pp. 1547-1553, 2009.

[8] A. Sezgin and A. O. Atagün, "On operations of soft sets," Computers \& Mathematics with Applications, vol. 61, no. 5, pp. 1457-1467, 2011.

[9] N. Çağman and S. Enginoğlu, "Soft matrix theory and its decision making," Computers and Mathematics with Applications, vol. 59, no. 10, pp. 3308-3314, 2010.

[10] N. Çağman and S. Enginoğlu, "Soft set theory and uni-int decision making," European Journal of Operational Research, vol. 207, pp. 848-855, 2010.

[11] P. K. Maji, R. Biswas, and A. R. Roy, "Fuzzy soft sets," Journal of Fuzzy Mathematics, vol. 9, pp. 589-602, 2001.

[12] P. K. Maji, R. Biswas, and A. Roy, "Intuitionistic fuzzy soft sets," Journal of Fuzzy Mathematics, vol. 9, pp. 677-692, 2001.

[13] K. Atanassov and G. Gargov, "Interval valued intuitionistic fuzzy sets,” Fuzzy Sets and Systems, vol. 31, no. 3, pp. 343-349, 1989.

[14] H. Garg and K. Kumar, "A novel possibility measure to interval-valued intuitionistic fuzzy set using connection number of set pair analysis and its applications," Neural Computing and Applications, vol. 32, no. 8, pp. 3337-3348, 2020.
[15] X. Yang, T. Y. Lin, J. Yang, Y. Li, and D. Yu, "Combination of interval-valued fuzzy set and soft set," Computers \& Mathematics with Applications, vol. 58, no. 3, pp. 521-527, 2009.

[16] Y. Jiang, Y. Tang, Q. Chen, H. Liu, and J. Tang, "Intervalvalued intuitionistic fuzzy soft sets and their properties," Computers \& Mathematics with Applications, vol. 60, no. 3, pp. 906-918, 2010.

[17] X. Ma, N. Sulaiman, and M. Rani, “Applications of intervalvalued intuitionistic fuzzy soft sets in a decision making problem," in Proceedings of the International Conference on Software Engineering and Computer Systems, pp. 642-651, Springer, Kuantan, Malaysia, June 2011.

[18] C. L. Hwang and K. Yoon, Multiple Attribute Decision Making: Methods and Applications, Springer, Berlin Germany, 1981.

[19] M. Zulqarnain, F. Dayan, and M. Saeed, "TOPSIS analysis for the prediction of diabetes based on general characteristics of humans," International Journal of Pharmaceutical Sciences and Research, vol. 9, no. 7, pp. 2932-2939, 2018.

[20] A. Sarkar, "A TOPSIS method to evaluate the technologies," International Journal of Quality \& Reliability Management, vol. 31, no. 1, pp. 2-13, 2013.

[21] R. M. Zulqarnain, S. Abdal, B. Ali et al., "Selection of medical clinic for disease diagnosis by using TOPSIS method," International Journal of Pharmaceutical Sciences Review and Research, vol. 61, no. 1, pp. 22-27, 2020.

[22] R. M. Zulqarnain, M. Saeed, N. Ahmad, F. Dayan, and B. Ahmad, "Application of TOPSIS method for decision making," International Journal of Scientific Research in Mathematical and Statistical Sciences, vol. 7, no. 2, pp. 76-81, 2020.

[23] C.-T. Chen, "Extensions of the TOPSIS for group decisionmaking under fuzzy environment," Fuzzy Sets and Systems, vol. 114, no. 1, pp. 1-9, 2000.

[24] L. Dymova, P. Sevastjanov, and A. Tikhonenko, "An approach to the generalization of fuzzy TOPSIS method," Information Sciences, vol. 238, pp. 149-162, 2013.

[25] M. Zulqarnain and F. Dayan, "Choose best criteria for decision making via fuzzy topsis method," Mathematics and Computer Science, vol. 2, no. 6, pp. 113-119, 2017.

[26] A. Y. Yayla, A. Yildiz, and O. Ahmet, "Fuzzy TOPSIS method in supplier selection and application in the garment industry," Fibres and Textiles in Eastern Europe, vol. 93, no. 4, pp. 20-23, 2012.

[27] A. Biswas and S. Kumar, "An integrated TOPSIS approach to MADM with interval-valued intuitionistic fuzzy settings," in Advanced Computational and Communication Paradigms, pp. 533-544, Springer, Singapore, 2018.

[28] M. Zulqarnain and F. Dayan, "Selection of best alternative for an automotive company by intuitionistic fuzzy TOPSIS method," International Journal of Scientific \& Technology Research, vol. 6, no. 10, pp. 126-132, 2017.

[29] H. Garg and K. Kumar, "Linguistic interval-valued Atanassov intuitionistic fuzzy sets and their applications to group decision making problems," IEEE Transactions on Fuzzy Systems, vol. 27, no. 12, pp. 2302-2311, 2019.

[30] H. Garg and R. Arora, "Generalized and group-based generalized intuitionistic fuzzy soft sets with applications in decision-making," Applied Intelligence, vol. 48, no. 2, pp. 343-356, 2018.

[31] H. Garg and R. Arora, Generalized Maclaurin Symmetric Mean Aggregation Operators Based on Archimedean T-Norm of the Intuitionistic Fuzzy Soft Set Information, Artifcial Intelligence Review, Netherlands, 2020.

[32] T. Mahmood and Z. Ali, "Entropy measure and TOPSIS method based on correlation coefficient using complex q-rung orthopair 
fuzzy information and its application to multi-attribute decision making," Soft Computing, vol. 11, pp. 1-27, 2020.

[33] T. Gerstenkorn and J. Mańko, "Correlation of intuitionistic fuzzy sets,” Fuzzy Sets and Systems, vol. 44, no. 1, pp. 39-43, 1991.

[34] C. Yu, "Correlation of fuzzy numbers," Fuzzy Sets and Systems, vol. 55, no. 3, pp. 303-307, 1993.

[35] D.-A. Chiang and N. P. Lin, "Correlation of fuzzy sets," Fuzzy Sets and Systems, vol. 102, no. 2, pp. 221-226, 1999.

[36] W.-L. Hung and J.-W. Wu, "Correlation of intuitionistic fuzzy sets by centroid method," Information Sciences, vol. 144, no. 1-4, pp. 219-225, 2002.

[37] H. Bustince and P. Burillo, "Correlation of interval-valued intuitionistic fuzzy sets," Fuzzy Sets and Systems, vol. 74, no. 2, pp. 237-244, 1995.

[38] D. H. Hong, "A note on correlation of interval-valued intuitionistic fuzzy sets," Fuzzy Sets and Systems, vol. 95, no. 1, pp. 113-117, 1998.

[39] H. B. Mitchell, "A correlation coefficient for intuitionistic fuzzy sets," International Journal of Intelligent Systems, vol. 19, no. 5, pp. 483-490, 2004.

[40] H. Garg and R. Arora, "TOPSIS method based on correlation coefficient for solving decision-making problems with intuitionistic fuzzy soft set information," AIMS Mathematics, vol. 5, no. 4, pp. 2944-2966, 2020.

[41] H.-L. Huang and Y. Guo, "An improved correlation coefficient of intuitionistic fuzzy sets," Journal of Intelligent Systems, vol. 28, no. 2, pp. 231-243, 2019.

[42] S. Singh, S. Sharma, and S. Lalotra, "Generalized correlation coefficients of intuitionistic fuzzy sets with application to MAGDM and clustering analysis," International Journal of Fuzzy Systems, vol. 22, no. 5, pp. 1582-1595, 2020.

[43] N. X. Thao, "A new correlation coefficient of the intuitionistic fuzzy sets and its application," Journal of Intelligent and Fuzzy Systems, vol. 35, no. 2, pp. 1959-1968, 2018.

[44] H. Garg and R. Arora, "Maclaurin symmetric mean aggregation operators based on t-norm operations for the dual hesitant fuzzy soft set," Journal of Ambient Intelligence and Humanized Computing, vol. 11, no. 1, pp. 375-410, 2020.

[45] C. Jana, G. Muhiuddin, and M. Pal, "Some Dombi aggregation of Q-rung orthopair fuzzy numbers in multiple-attribute decision making," International Journal of Intelligent Systems, vol. 34, no. 12, pp. 3220-3240, 2019.

[46] R. Arora and H. Garg, "Robust aggregation operators for multicriteria decision-making with intuitionistic fuzzy soft set environment," Scientia Iranica E, vol. 25, no. 2, pp. 931-942, 2018.

[47] S. Sudharsan and D. Ezhilmaran, "Weighted arithmetic average operator based on interval-valued intuitionistic fuzzy values and their application to multi criteria decision making for investment," Journal of Information and Optimization Sciences, vol. 37, no. 2, pp. 247-260, 2016.

[48] M. Imtiaz, M. Saqlain, and M. Saeed, "TOPSIS for multi criteria decision making in octagonal intuitionistic fuzzy environment by using accuracy function," Journal of New Theory, vol. 31, pp. 32-40, 2020.

[49] H. Wu and X. Su, "Group Generalized interval-valued intuitionistic fuzzy soft sets and their applications in decision making," Iranian Journal of Fuzzy Systems, vol. 14, no. 1, pp. 1-21, 2017.

[50] A. Mukherjee and S. Sarkar, "Similarity measures for intervalvalued intuitionistic fuzzy soft sets and its application in medical diagnosis problem," New Trends in Mathematical Sciences, vol. 2, no. 3, pp. 159-165, 2014. 